\title{
Unexpected formation of complex bridged tetrazoles via intramolecular 1,3-dipolar cycloaddition of 1,2-O-cyanoalkylidene derivatives of 3-azido-3-deoxy-D-allose
}

\author{
Marco Worch and Valentin Wittmann* \\ Fachbereich Chemie, Universität Konstanz, D-78457 Konstanz, Germany \\ Received 1 October 2007; accepted 23 October 2007
}

Presented at Eurocarb 14th Lübeck, Germany, September 2007

\begin{abstract}
An unexpected and interesting intramolecular side reaction occurred during the attempted synthesis of glycosyl cyanides upon treatment of 1-O-acetyl-3-azido-3-deoxyallose derivatives with TMSCN and different Lewis acids. Exo-1,2-O-cyanoalkylidene derivatives formed by neighboring group participation and attack of cyanide underwent, after Lewis-acid mediated isomerization to the endo-isomer, intramolecular azide-cyanide cycloaddition leading to the formation of tetrazoles embedded in bridged tetracyclic ring systems. The efficiency of cycloaddition is dependent on the ring structure of the sugar (pyranose or furanose). Of the studied molecules, 3-azido-1,2-O-cyanoethylidene-3-deoxy-allopyranose provides the most suitable scaffold for intramolecular [2+3] cycloaddition under exceptionally mild conditions. Our results highlight the capability of carbohydrates to act as scaffolds for the precise positioning of functional groups productive for a specific chemical reaction.
\end{abstract}

(C) 2007 Elsevier Ltd. All rights reserved.

Keywords: C-Glycosyl compounds; Cyanides; Cyanoethylidene; Cycloaddition; Neighboring group participation

\section{Introduction}

Tetrazoles are becoming an increasingly popular functionality. They can serve as bioisosteres of carboxylic acids, ${ }^{1,2}$ as precursors of nitrogen-containing heterocycles, and as lipophilic spacers in pharmaceuticals. Recently reported was their use as $\beta$-secretase inhibitors, ${ }^{3,4}$ Kv1.5 blockers, ${ }^{5}$ and as carboxylate bioisosteres of gabapentin and pregabalin. ${ }^{6,7}$ A potentially useful method for the preparation of 1,5-disubstituted tetrazoles is the $[2+3]$ cycloaddition of organic azides to nitriles. To date, however, only a few electron deficient nitriles are known that undergo this reaction in an intermolecular way under very forcing conditions. ${ }^{8-10}$ High yields have been reported for the reaction of sulfonyl and acyl cyanides with unhindered aliphatic azides by neat, thermal fusion. ${ }^{11,12}$ Intramolecular $[2+3]$ cyclo-

\footnotetext{
* Corresponding author. Tel.: +49 753188 4572; fax: +49 753188 4573; e-mail: mail@valentin-wittmann.de
}

addition reactions of organic azides to nitriles occur more readily. ${ }^{13-17}$ Still, they require high reaction temperatures to proceed and yields are with few exceptions $^{18}$ not satisfactory. In the course of our investigations toward the Lewis-acid promoted synthesis of glycosyl cyanides, we now observed an unexpected side reaction leading for the first time to complex bridged tetracyclic ring systems via 1,3-dipolar cycloaddition of intermediate cyanoethylidene derivatives of 3 -azidoallose even at room temperature.

The formation of cyanoethylidene compounds is a common side reaction in $\mathrm{C}$-glycosylation reactions. They are formed when ester protecting groups are used at $\mathrm{O}-2$ and the C-nucleophile is cyanide. ${ }^{19-21}$ Kochetkov and coworkers utilized cyanoethylidene compounds in $\mathrm{O}$ glycosylation reactions and showed that the $\mathrm{C}-\mathrm{C}$ bond between the dioxolane carbon and the carbon of the cyano group is relatively weak and can be cleaved with catalytic amounts of Lewis acids such as trityl perchlorate. $^{22-24}$ Myers et al. showed that cyanoethylidene 
compounds of mannose, glucose, and galactose can be converted to anomeric cyanides under acid promotion without an exogenous cyanide source. ${ }^{19}$ Intra- or intermolecular cycloaddition reactions of cyanoethylidene compounds have not been reported before.

\section{Results and discussion}

In the course of our program directed at the synthesis of sugar diamino acids (SDAs) as building blocks of oligosaccharide mimetics, ${ }^{25}$ we were interested in glycosyl cyanide 4 of 3-azido-3-deoxy-D-allopyranose (Scheme 1). Starting from 3-azido-3-deoxy-1,2:5,6-di- $O$-isopropylidene- $\alpha$-D-allofuranose $1^{26}$ we obtained the peracetylated allopyranose derivative $\mathbf{3}$ using two different pathways. When using $60 \%$ formic acid for the cleavage of the isopropylidene protecting groups of $\mathbf{1}$, we observed the formation of two different formylated side products, which could subsequently be converted with $\mathrm{NaOMe} / \mathrm{MeOH}$ to the completely deprotected 3-azido3-deoxy-D-allose 2 existing predominantly as $\beta$-pyra- nose. Treatment with acetic anhydride in pyridine afforded peracetylated $\beta$-pyranose 3 in $91 \%$ yield over 3 steps besides small amounts of the corresponding $\alpha$ - and $\beta$ furanose and $\alpha$-pyranose forms. Alternatively, stirring of 1 with $2 \mathrm{M} \mathrm{HCl}$ directly resulted in the formation of $\mathbf{2}$, which was again peracetylated under the same conditions. In this case, however, the yield of $\beta$-pyranose 3 was somewhat lower ( $70 \%$ over 2 steps). For both reaction pathways the amounts of $\alpha$-pyranose and the $\alpha$ - and $\beta$-furanose forms were negligible and completely separable by HPLC purification.

To synthesize glycosyl cyanide $\mathbf{4}$, peracetate $\mathbf{3}$ was treated with excess of trimethylsilyl cyanide (TMSCN) and equimolar amounts of tin tetrachloride as Lewis acid promoter. However, we were not able to find even traces of the desired $C$-glycosyl compounds under these conditions. Instead, we observed a new compound that, after extensive analysis by NMR and IR spectroscopy and mass spectrometry, turned out to be tetrazole $\mathbf{6}$. As an intermediate, exo-cyanoethylidene derivative $\mathbf{5}$ was identified (see below). Figure 1 depicts characteristic NMR data of 5 and $\mathbf{6}$. Tetrazole $\mathbf{6}$ shows a ${ }^{13} \mathrm{C}$ NMR

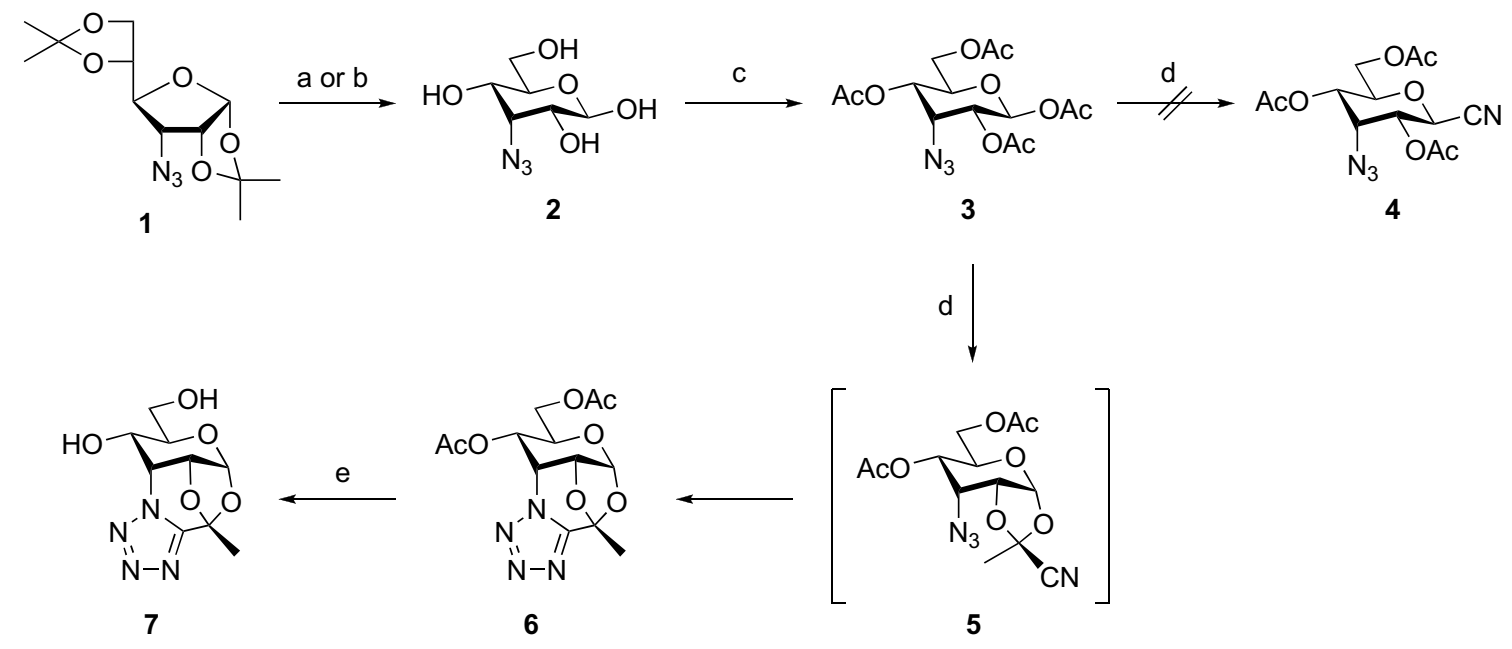

Scheme 1. Reagents and conditions: (a) (i) $60 \% \mathrm{HCOOH}, 16$ h; (ii) $\mathrm{NaOMe}, \mathrm{MeOH}, 2 \mathrm{~h}$; (b) $2 \mathrm{M} \mathrm{HCl}, 6$ h; (c) Ac 2 O, pyr, overnight, $91 \%$ [(a) + (c)], $70 \%\left[\left(\right.\right.$ b) + (c)]; (d) 5 equiv TMSCN, 1.2 equiv $\mathrm{SnCl}_{4}, \mathrm{CH}_{2} \mathrm{Cl}_{2}, 60{ }^{\circ} \mathrm{C}, 65 \%$; (e) $\mathrm{NaOMe}, \mathrm{MeOH}, 14 \mathrm{~h}, 84 \%$.

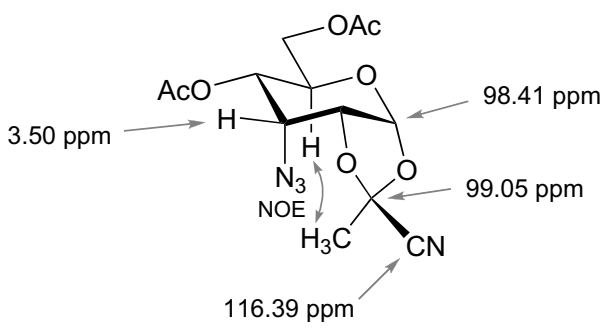

5

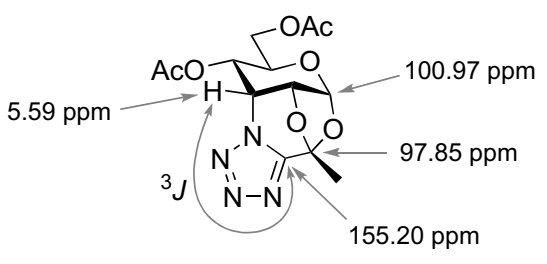

6

Figure 1. Comparison of significant ${ }^{1} \mathrm{H}$ and ${ }^{13} \mathrm{C}$ NMR chemical shifts of compounds 5 and $\mathbf{6}$. Also indicated are an NOE between $\mathrm{H}-5$ and the cyanoethylidene methyl group of 5 indicative for the exo-configuration and a ${ }^{3} J_{\mathrm{C}, \mathrm{H}}$ long-range coupling observed in a ${ }^{1} \mathrm{H}$, ${ }^{13} \mathrm{C} \mathrm{HMBC} \mathrm{NMR} \mathrm{spectrum}$ characteristic for tetrazole $\mathbf{6}$. 
resonance at $155.20 \mathrm{ppm}$, which is typical for a tetrazole carbon ${ }^{27,28}$ and a strong signal in a ${ }^{1} \mathrm{H},{ }^{13} \mathrm{C}$ HMBC NMR spectrum due to a ${ }^{3} J_{\mathrm{C}, \mathrm{H}}$ long-range coupling between the tetrazole carbon and H-3. In addition, the cyanide resonance in a ${ }^{13} \mathrm{C}$ NMR spectrum and the nitrile and azide resonances in an IR spectrum (between 2100 and $2130 \mathrm{~cm}^{-1}$, medium intensity) were missing for $\mathbf{6}$. Tetrazole 6 could be deacetylated with $\mathrm{NaOMe}$ in methanol leading to the formation of diol 7 in $84 \%$ yield. Further investigations of the tetrazole formation using different solvents and Lewis acids at different temperatures revealed that 6 emerged as the major product in every case and no anomeric cyanide 4 was formed. Table 1 gives an overview over some of the experiments we carried out.

To isolate and characterize cyanoethylidene intermediate 5, we reacted 3 with TMSCN (5 equiv) and catalytic amounts of Lewis acid $\left(0.2\right.$ equiv $\left.\mathrm{SnCl}_{4}\right)$ at room temperature. After column chromatography, $\mathbf{5}$ was isolated in $22 \%$ yield beside tetrazole $6(33 \%)$ and starting material $3(12 \%)$. NOE experiments carried out with cyanoethylidene derivative 5 using a mixing time of 300 ms revealed an NOE between the dioxolane methyl group and $\mathrm{H}-5$ indicating that $\mathbf{5}$ is the exo-cyanoethylidene isomer. Pure 5 was treated with TMSCN and $\mathrm{SnCl}_{4}$ or with $\mathrm{SnCl}_{4}$ alone. In both cases we observed that tetrazole $\mathbf{6}$ was formed as the only product beside some decomposition. Addition of TMSCN to a solution of
$\mathrm{SnCl}_{4}$ and 5 in $\mathrm{CH}_{2} \mathrm{Cl}_{2}$ increased the speed of reaction but had no effect on the yield, which was approximately $70 \%$. We always had to use at least equimolar amounts of Lewis acid to effect complete consumption of starting material 5.

Scheme 2 shows the proposed mechanism by which tetrazole $\mathbf{6}$ is formed. Starting from 3, neighboring group participation leads to acetoxonium ion $\mathbf{8}$ that can be attacked by cyanide either from the exo or the endo side. Formation of exo-cyanoethylidene $\mathbf{5}$ is expected to be kinetically favored due to an attack from the sterically less hindered convex side of bicyclic $\mathbf{8}$. Formation of exo-cyanoethylidenes has been described earlier by others to be favored over the formation of endo-cyanoethylidenes ${ }^{22-24,29}$ and also corresponds to the preferred formation of exo-isomeric forms of ortho esters. $^{30}$ Under the reaction conditions, however, cyanide attack is reversible giving also access to endocyanoethylidene 9. Proper spatial orientation of azido and cyano groups within endo-cyanoethylidene 9 obviously leads to a fast irreversible intramolecular cycloaddition forming tetrazole 6 . In agreement with a fast cycloaddition step is the observation that we were never able to detect any of the endo-cyanoethylidene isomer $\mathbf{9}$.

To investigate whether tetrazole formation also occurs with protecting groups that are known to reduce the amount of cyanoethylidene formation, ${ }^{31,32}$ we

Table 1. Results of reactions of 3-azido-3-deoxy-allopyranose 3 with 5 equiv of TMSCN under varying conditions

\begin{tabular}{lllllll}
\hline Solvent & Lewis acid & $T\left({ }^{\circ} \mathrm{C}\right)$ & Time & Yield $\mathbf{5}(\%)$ & Yield $\mathbf{6}(\%)$ & Recovered 3 $(\%)$ \\
\hline $\mathrm{CH}_{2} \mathrm{Cl}_{2}$ & 0.4 equiv $\mathrm{SnCl}_{4}$ & 60 & $5 \mathrm{~h}$ & 14 & 8 & 52 \\
$\mathrm{CH}_{2} \mathrm{Cl}_{2}$ & 1.2 equiv $\mathrm{SnCl}_{4}$ & 60 & $2 \mathrm{~h}$ & - & 65 & - \\
$\mathrm{CH}_{2} \mathrm{Cl}_{2}$ & 0.2 equiv $\mathrm{SnCl}_{4}$ & 20 & $18 \mathrm{~h}$ & 22 & 9 & 65 \\
$\mathrm{H}_{3} \mathrm{CNO}_{2}$ & 0.9 equiv $\mathrm{BF}_{3} \cdot \mathrm{OEt}_{2}$ & 20 & $1 \mathrm{~h} 20 \mathrm{~min}$ & 8 & 60 & - \\
$\mathrm{H}_{3} \mathrm{CNO}_{2}$ & 1.2 equiv $\mathrm{BF}_{3} \cdot \mathrm{OEt}_{2}$ & 60 & $20 \mathrm{~min}$ & - & 35 & - \\
\hline
\end{tabular}

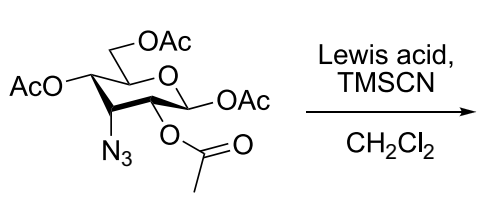

3
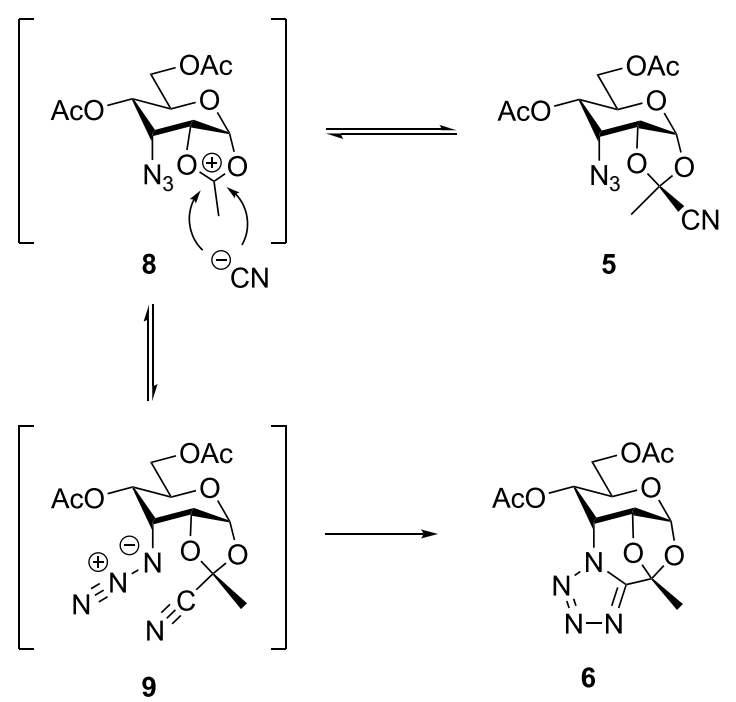

Scheme 2. Proposed mechanism for intramolecular tetrazole formation. 


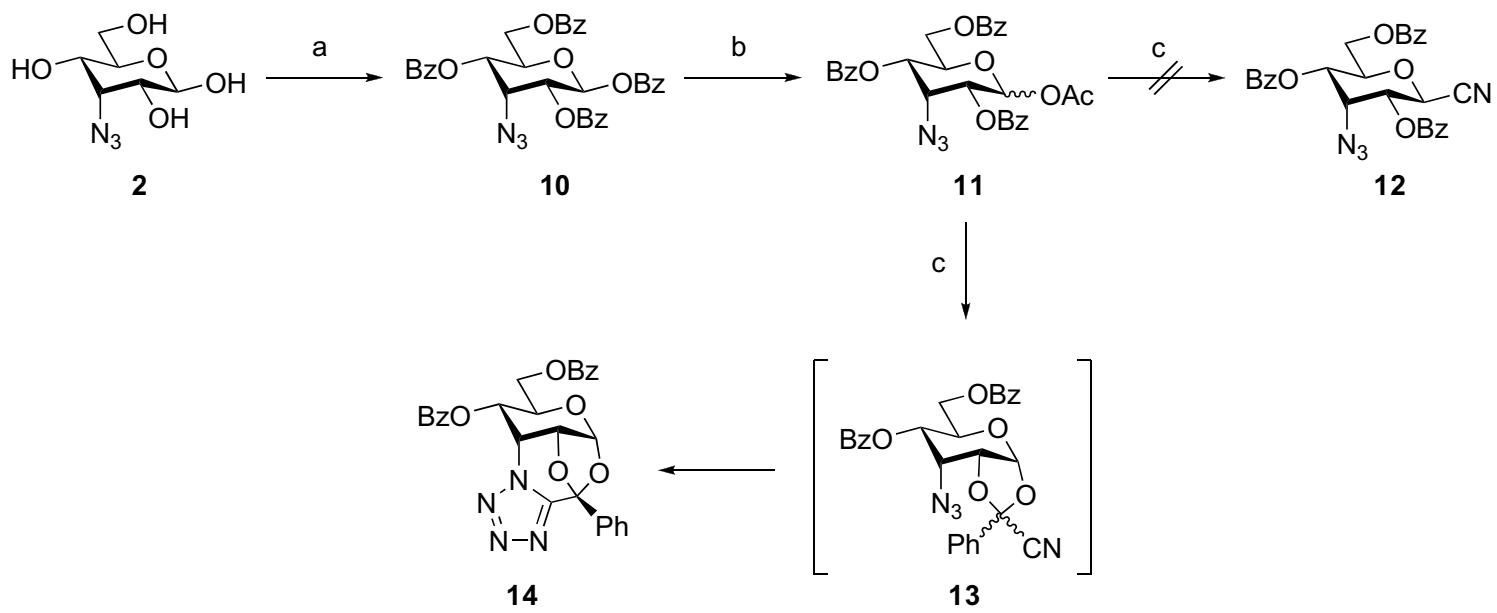

Scheme 3. Reagents and conditions: (a) $\mathrm{BzCl}$, pyr, $24 \mathrm{~h}, 77 \%$; (b) (i) $\mathrm{H}_{2} \mathrm{~N}-\mathrm{NH}_{2} \cdot \mathrm{HOAc}, \mathrm{DMF}, 50{ }^{\circ} \mathrm{C}, 3 \mathrm{~h}$; (ii) $\mathrm{Ac}{ }_{2} \mathrm{O}, \mathrm{pyr}, 12 \mathrm{~h}, 41 \%$ ( $2 \mathrm{steps}$ ); (c) 10 equiv TMSCN, 1.6 equiv $\mathrm{BF}_{3} \cdot \mathrm{OEt}_{2}, \mathrm{H}_{3} \mathrm{CNO}_{2}, 50 \%$.

decided to introduce a benzoyl group as protection for O-2. Azidoallose 2 was perbenzoylated with benzoyl chloride in pyridine to give $\mathbf{1 0}$ in a yield of $77 \%$ (Scheme $3)$. Experiments to react $\mathbf{1 0}$ directly with TMSCN and $\mathrm{SnCl}_{4}$ failed. Obviously, the anomeric benzoyl group cannot act as a leaving group under these conditions and starting material was recovered completely. Anomeric deprotection of $\mathbf{1 0}$ by treatment with hydrazinium acetate for $3 \mathrm{~h}$ at $50^{\circ} \mathrm{C}$ and subsequent acetylation with pyridine $/ \mathrm{Ac}_{2} \mathrm{O}$ led to the formation of $\mathbf{1 1}$ as a mixture of anomers $(\alpha: \beta=1: 2.9)$ in $41 \%$ yield over 2 steps. Compound 11 was treated with $\mathrm{TMSCN}$ and $\mathrm{BF}_{3} \cdot \mathrm{OEt}_{2}$ in nitromethane and, again, the formation of a tetrazole (14) was observed $(50 \%$ yield). Glycosyl cyanide 12 was not found. In this case, the yield of $\mathbf{1 4}$ dropped to $12 \%$ when dichloromethane was used instead of nitromethane.

Since we observed tetrazole formation even with a benzoyl group at $\mathrm{O}-2$, we wanted to know if azidoallose acts as a template for the intramolecular cycloaddition reaction only in its pyranose constitution or if this cyclization can also be achieved with the corresponding acetylated furanose (17). To synthesize 17, furanose 1 was treated with a solution of $\mathrm{HCl}$ in methanol (Scheme 4). After stirring for $48 \mathrm{~h}$, the formation of a mixture of methyl glycosides $\mathbf{1 5}$ and $\mathbf{1 6}$ was observed. Quenching of the reaction mixture with triethylamine and subse- quent chromatographic purification afforded the desired triol 15 in a yield of $56 \%$ and 5,6-O-isopropylidene derivative 16 in $34 \%$ yield. Neutralization of the reaction mixture was necessary, as direct solvent removal gave small amounts of the corresponding methyl $\alpha$ - and $\beta$ D-3-azido-3-deoxy-allopyranosides, which were not separable from 15 by column chromatography. Conversion of 16 into 15 was possible using the same conditions as before, thus raising the overall yield of $\mathbf{1 5}$ to $78 \%$. Subsequently 15 was converted into the peracetylated furanose 17 in 2 steps in a yield of $83 \%$ (Scheme 5).

Treatment of $\mathbf{1 7}$ with 5 equiv of TMSCN and 1.1 equiv of $\mathrm{SnCl}_{4}$ in $\mathrm{CH}_{2} \mathrm{Cl}_{2}$ at $60{ }^{\circ} \mathrm{C}$ led to complete consumption of the starting material and a product mixture of glycosyl cyanide $18(27 \%)$ and tetrazole $19(52 \%)$ was isolated. NMR studies of this reaction in $\mathrm{CD}_{2} \mathrm{Cl}_{2}$ revealed that two major intermediates were formed. To isolate these intermediates, 17 was treated with 5 equiv of TMSCN and only 0.5 equiv of $\mathrm{SnCl}_{4}$ at room temperature. Under these conditions we were able to isolate the two intermediates $20(19 \%)$ and $21(6 \%)$ by HPLC beside 18 (19\%) and 19 (37\%). Analysis by NMR spectroscopy revealed that the intermediates were the two isomeric cyanoethylidenes (Scheme 1), which were formed in an exo/endo ratio of approximately 3:1. Since the endo-cyanoethylidene derivative 21 could be isolated in the allofuranose case, we conclude that

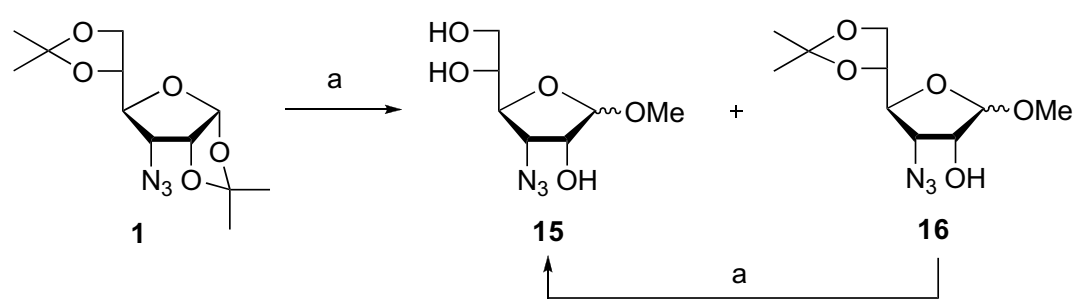

Scheme 4. Reagents and conditions: (a) (i) $\mathrm{MeOH}, \mathrm{HCl}, 48$ h; (ii) $\mathrm{Et}_{3} \mathrm{~N}, \mathrm{pH} 7,78 \%$. 


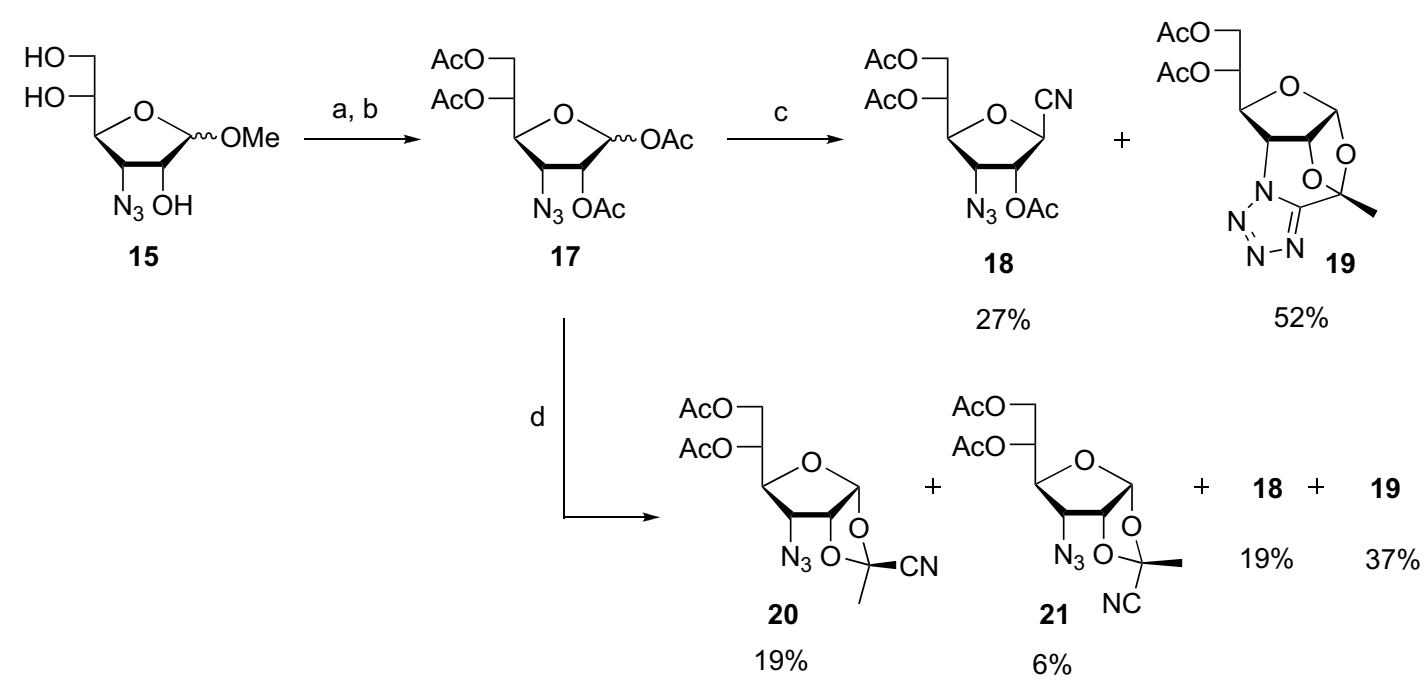

Scheme 5. Reagents and conditions: (a) $\mathrm{Ac}_{2} \mathrm{O}$, pyr, $12 \mathrm{~h}$; (b) 75 equiv $\mathrm{HOAc}, 12$ equiv $\mathrm{Ac}_{2} \mathrm{O}, 6$ equiv concd $\mathrm{H}_{2} \mathrm{SO}_{4}, 30 \mathrm{~min}, 83 \%$ ( 2 steps); (c) 5 equiv TMSCN, 1.1 equiv $\mathrm{SnCl}_{4}, \mathrm{CH}_{2} \mathrm{Cl}_{2}, 60^{\circ} \mathrm{C}$; (d) 5 equiv TMSCN, 0.5 equiv $\mathrm{SnCl}_{4}, \mathrm{CH}_{2} \mathrm{Cl}_{2}, \mathrm{rt}$.

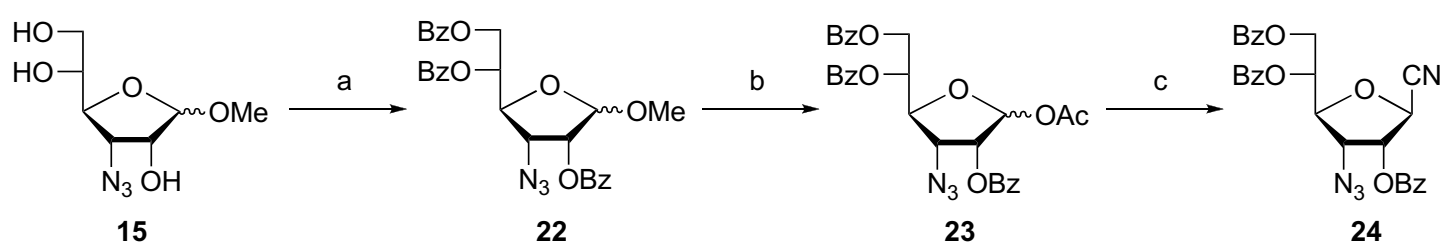

Scheme 6. Reagents and conditions: (a) $\mathrm{BzCl}$, pyr, $24 \mathrm{~h}, 89 \%$; (b) 70 equiv $\mathrm{HOAc}, 10$ equiv $\mathrm{Ac}_{2} \mathrm{O}, 5$ equiv concd $\mathrm{H}_{2} \mathrm{SO}_{4}, 36$ min, $90 \%$; (c) 5 equiv TMSCN, 1.1 equiv $\mathrm{SnCl}_{4}, \mathrm{CH}_{2} \mathrm{Cl}_{2}, 4 \mathrm{~h}, 60{ }^{\circ} \mathrm{C}, 65 \%$.

in this case the spatial orientation of the cyano and azido groups is not as favorable for a subsequent cycloaddition as in the allopyranose case, leading to a slower rate of the tetrazole formation.

The observation, that glycosyl cyanide $\mathbf{1 8}$ was formed beside tetrazole $\mathbf{1 9}$ in the allofuranose case, led us to the speculation that the introduction of a benzoyl group at $\mathrm{O}-2$ this time could lead to the formation of the anomeric cyanide as the major product. To prove this, $\mathbf{1 5}$ was perbenzoylated with benzoyl chloride in pyridine to yield 22 in $89 \%$ yield (Scheme 6). Subsequently, the anomeric acetyl group was introduced by acetolysis of 22 with concd $\mathrm{H}_{2} \mathrm{SO}_{4}, \mathrm{HOAc}$ and $\mathrm{Ac}_{2} \mathrm{O}$ to give 23 in $90 \%$ yield. Treatment of $\mathbf{2 3}$ with 5 equiv of TMSCN and 1.1 equiv of $\mathrm{SnCl}_{4}$ in $\mathrm{CH}_{2} \mathrm{Cl}_{2}$ led to the formation of the glycosyl cyanide $\mathbf{2 4}$ as the major product (65\%), as expected. This time, we did not observe any tetrazole formation.

\section{Conclusion}

In summary, we investigated an unexpected and interesting side reaction during the attempted synthesis of glycosyl cyanides by treatment of 1-O-acetyl-3-azido-3deoxyallose derivatives with TMSCN and different
Lewis acids. exo-1,2-O-Cyanoalkylidene derivatives formed by neighboring group participation and attack of cyanide underwent, after Lewis-acid mediated isomerization to the endo-isomer, intramolecular azidecyanide cycloaddition leading to the formation of tetrazoles embedded in bridged tetracyclic ring systems. The efficiency of cycloaddition is dependent on the ring structure of the sugar. Of the studied molecules, 3-azido1,2- $O$-cyanoethylidene-3-deoxy-allopyranose provides the most suitable scaffold for intramolecular $[2+3]$ cycloaddition under exceptionally mild conditions. Tetrazole formation is even observed with a 2-O-benzoyl protecting group that is known to give diminished amounts of cyanoalkylidene derivatives. In the case of 3-azido-3-deoxy-allofuranose, production of a glycosyl cyanide was observed beside tetrazole formation when $\mathrm{O}-2$ was acetylated indicating a less precise positioning of azide and cyanide. Tetrazole formation can be completely prevented in the furanose case by introducing a benzoyl group at $\mathrm{O}-2$. In this case, the glycosyl cyanide is the major product.

It has been shown that carbohydrates can function as scaffolds for the spatially defined presentation of pharmacophores. ${ }^{33}$ Our results highlight the capability of carbohydrates to act as scaffolds for the precise positioning of functional groups productive for a specific 
chemical reaction. It is conceivable that pyranose forms of other sugars, having a similar stereochemical structure at C-2 and C-3, like gulose or ribose, could also act as scaffolds for the same reaction.

\section{Experimental}

\subsection{General methods}

All solvents were dried and freshly distilled using standard procedures. ${ }^{34}$ Organic solutions were concentrated under diminished pressure with bath temperatures $<40{ }^{\circ} \mathrm{C}$. Cation exchange resin Amberlite IR-120 $\left(\mathrm{H}^{+}\right)$ was pre-washed with dry $\mathrm{MeOH}$ before use. Thin-layer chromatography (TLC) was performed on aluminumbacked, pre-coated silica gel plates (Silica Gel $60 \mathrm{~F}_{254}$, E. Merck). Silica Gel 60 (200-400 mesh) was used for column chromatography. MALDI-TOF mass spectra were recorded on a Bruker Biflex III spectrometer in positive, linear mode. High resolution ESI mass spectra were recorded on an Apex II spectrometer using an Apollo ESI source. FT-IR spectra were recorded on a Perkin Elmer System 2000 FT-IR spectrometer. NMR spectra were recorded either with a Bruker DRX 600 $\left(600 \mathrm{MHz}\right.$ for ${ }^{1} \mathrm{H}$ and $150 \mathrm{MHz}$ for ${ }^{13} \mathrm{C}$ ) or a Bruker AC $250\left(250 \mathrm{MHz}\right.$ for ${ }^{1} \mathrm{H}$ and $62.5 \mathrm{MHz}$ for $\left.{ }^{13} \mathrm{C}\right)$ spectrometer. Resonance assignments were made by the aid of COSY, HSQC, HMQC, HMBC, and NOESY experiments, when necessary. Chemical shifts are reported relative to $\mathrm{CHCl}_{3}\left(\delta_{\mathrm{H}} 7.26, \delta_{\mathrm{C}} 77.0 \mathrm{ppm}\right)$, DMSO $\left(\delta_{\mathrm{H}} 2.50, \delta_{\mathrm{C}} 39.43 \mathrm{ppm}\right)$, or $\mathrm{MeOH}\left(\delta_{\mathrm{C}} 49.50 \mathrm{ppm}\right)$ as internal standard for $\mathrm{D}_{2} \mathrm{O}$. In the case of $\alpha / \beta$ and endo/exo mixtures, elemental analysis was carried out using a mixture of both isomers, even when the isomers were separated for the recording of NMR spectra.

\subsection{3-Azido-3-deoxy- $\beta$-D-allopyranose (2)}

A $0.4 \mathrm{M}$ soln of $\mathrm{NaOMe}$ in $\mathrm{MeOH}(2.6 \mathrm{~mL}, 0.1$ equiv) was added to a stirred soln of $3(3.9 \mathrm{~g}, 10.45 \mathrm{mmol})$ in dry $\mathrm{MeOH}(20 \mathrm{~mL})$. After $6 \mathrm{~h}$, ion exchange resin Amberlite IR-120 ( $\mathrm{H}^{+}$form) was added until the $\mathrm{pH}$ was 7. Evaporation to dryness afforded $\mathbf{2}$ as a white wax-like solid $(1.93 \mathrm{~g}, 9.4 \mathrm{mmol}, 90 \%)$. Beside the $\beta$ pyranose form, minor signals from the $\alpha$-pyranose and the $\alpha$ - and $\beta$-furanose forms were detected in the NMR spectrum of compound 2 in a ratio of $\beta_{\mathrm{Pyr}}$ : $\alpha_{\mathrm{Pyr}}$ : $\beta_{\text {Fur }}: \alpha_{\text {Fur }}=100: 4: 3:<1$. These minor products were not completely characterized.

$R_{\mathrm{f}} 0.33(\mathrm{EtOAc} / \mathrm{MeOH}) .{ }^{1} \mathrm{H}$ NMR $\left(\mathrm{D}_{2} \mathrm{O}, 250 \mathrm{MHz}\right)$ : $\delta 4.76\left(\mathrm{dd}, 1 \mathrm{H}, J_{1,2} 8.2 \mathrm{~Hz}, \mathrm{H}-1\right), 4.19\left(\mathrm{t}, 1 \mathrm{H}, J_{2,3} \approx J_{3,4}\right.$ $3.5 \mathrm{~Hz}, \mathrm{H}-3), 3.75-3.82$ (m, 2H, H-6, H-4), 3.58-3.69 (m, $2 \mathrm{H}, \mathrm{H}-5, \mathrm{H}-6$ ), 3.51 (dd, $\left.1 \mathrm{H}, J_{2,3} 3.5 \mathrm{~Hz}, \mathrm{H}-2\right) ;{ }^{13} \mathrm{C}$ NMR $\left(\mathrm{D}_{2} \mathrm{O}+2\right.$ drops $\left.\mathrm{MeOH}-d_{4}, 150 \mathrm{MHz}\right): \delta 94.97$ (C-1), 75.56 (C-5), 72.11 (C-2), 68.00 (C-3), 67.93
(C-4), 62.33 (C-6). MALDIMS (positive mode, DHB, $\mathrm{MeCN} / \mathrm{H}_{2} \mathrm{O}$ 1:1) $\mathrm{m} / \mathrm{z}$ calcd for $\mathrm{C}_{6} \mathrm{H}_{11} \mathrm{~N}_{3} \mathrm{O}_{5}: 228.1$ $[\mathrm{M}+\mathrm{Na}]^{+}$. Found: 228.0. Anal. Calcd for $\mathrm{C}_{6} \mathrm{H}_{11} \mathrm{~N}_{3} \mathrm{O}_{5}$. $0.75 \mathrm{H}_{2} \mathrm{O}: \mathrm{C}, 32.95 ; \mathrm{H}, 5.76 ; \mathrm{N}, 19.22$. Found: $\mathrm{C}$, 33.04; H, 5.71; N, 19.25 .

\subsection{1,2,4,6-Tetra- $O$-acetyl-3-azido-3-deoxy- $\beta$-D-allo- pyranose (3)}

Compound 3 was synthesized in two different ways.

(a) Compound 1 (5.3 g, $18.57 \mathrm{mmol}$ ) was suspended in $60 \%$ formic acid $(180 \mathrm{~mL})$ and stirred at room temperature for $16 \mathrm{~h}$. The solvent was removed under diminished pressure and the completely deprotected product was separated from formylated side product by column chromatography (EtOAc). The side product was then dissolved in $\mathrm{MeOH}(70 \mathrm{~mL})$ and a $0.4 \mathrm{M}$ soln of $\mathrm{NaOMe}$ in $\mathrm{MeOH}(2.15 \mathrm{~mL})$ was added. After $2 \mathrm{~h}$ ion exchange resin Amberlite IR-120 $\left(\mathrm{H}^{+}\right.$form) was added until the soln showed a $\mathrm{pH}$ of 7 . The solvent was removed under diminished pressure and the white crystalline residue was combined with the completely deprotected product. Combined products were dissolved in pyridine $(260 \mathrm{~mL})$ followed by the addition of $\mathrm{Ac}_{2} \mathrm{O}$ $(130 \mathrm{~mL})$ and stirred overnight. Solvent removal under diminished pressure followed by chromatographic purification (4:1 petroleum ether-EtOAc) afforded 3 as a colorless oil (6.31 g, $16.91 \mathrm{mmol}, 91 \%)$.

(b) Compound 1 (2.18 g, $7.64 \mathrm{mmol})$ was dissolved under ultrasonic agitation in $2 \mathrm{M} \mathrm{HCl}(76.4 \mathrm{~mL})$ and stirred for $6 \mathrm{~h}$ at room temperature. The solvent was removed under diminished pressure. The remaining dark brown oil was redissolved in pyridine $(110 \mathrm{~mL})$ under ultrasonic agitation. Subsequently, $\mathrm{Ac}_{2} \mathrm{O}(55 \mathrm{~mL})$ was added and the soln was stirred overnight. Solvent removal under diminished pressure followed by chromatographic purification (4:1 petroleum ether-EtOAc) afforded 3 as a colorless oil $(2.0 \mathrm{~g}, 5.35 \mathrm{mmol}, 70 \%)$.

In both cases (a) and (b) minor signals from the $\alpha$ pyranose and the $\alpha$ - and $\beta$-furanose forms were detected in the NMR spectrum of compound 3.

$R_{\mathrm{f}} 0.26$ (3:1 petroleum ether-EtOAc). IR (film): $v 2109$ $\left(\mathrm{N}_{3}\right), 1751 \mathrm{~cm}^{-1}(\mathrm{CO}) .{ }^{1} \mathrm{H}$ NMR $\left(\mathrm{CDCl}_{3}, 250 \mathrm{MHz}\right): \delta$ $5.96\left(\mathrm{~d}, 1 \mathrm{H}, J_{1,2} 8.5 \mathrm{~Hz}, \mathrm{H}-1\right), 4.92-4.99(\mathrm{~m}, 2 \mathrm{H}, \mathrm{H}-2$, $\mathrm{H}-4), 4.47\left(\mathrm{t}, 1 \mathrm{H}, J_{2,3} \approx J_{3,4} 3.4 \mathrm{~Hz}, \mathrm{H}-3\right), 4.29(\mathrm{dd}$, $\left.1 \mathrm{H}, J_{5,6} 4.2 \mathrm{~Hz}, J_{6,6^{\prime}} 12.3 \mathrm{~Hz}, \mathrm{H}-6\right), 4.10-4.21(\mathrm{~m}, 2 \mathrm{H}$, $\left.J_{5,6^{\prime}} 1.9 \mathrm{~Hz}, \mathrm{H}-5, \mathrm{H}-6^{\prime}\right), 2.13\left(\mathrm{~s}, 3 \mathrm{H}, \mathrm{CH}_{3} \mathrm{CO}\right), 2.12(\mathrm{~s}$, $\left.3 \mathrm{H}, \mathrm{CH}_{3} \mathrm{CO}\right), 2.11\left(\mathrm{~s}, 3 \mathrm{H}, \mathrm{CH}_{3} \mathrm{CO}\right), 2.08(\mathrm{~s}, 3 \mathrm{H}$, $\left.\mathrm{CH}_{3} \mathrm{CO}\right) ;{ }^{13} \mathrm{C}$ NMR $\left(\mathrm{CDCl}_{3}, 150 \mathrm{MHz}\right): \delta 170.57$ (CO), 169.32 (CO), 169.21 (CO), 168.90 (CO), 89.81 (C-1), $70.88\left(\mathrm{C}-5_{\beta}\right), 69.30$ (C-2), 66.87 (C-4), 61.82 (C6), 60.79 (C-3), $20.82\left(\mathrm{CH}_{3} \mathrm{CO}\right), 20.67\left(\mathrm{CH}_{3} \mathrm{CO}\right), 20.48$ $\left(\mathrm{CH}_{3} \mathrm{CO}\right), 20.45\left(\mathrm{CH}_{3} \mathrm{CO}\right)$. MALDIMS (positive mode, DHB, dioxane) $m / z$ calcd for $\mathrm{C}_{14} \mathrm{H}_{19} \mathrm{~N}_{3} \mathrm{O}_{9}: 396.2$ $[\mathrm{M}+\mathrm{Na}]^{+}$. Found: 396.2. Anal. Calcd: C, 45.04; H, 5.13; N, 11.26. Found: C, 45.24; H, 5.23; N, 11.17. 
4.4. 4,6-Di- $O$-acetyl-3-azido-3-deoxy-1,2-O-(1-exo-cyanoethylidene)- $\alpha$-D-allopyranose (5)

Trimethylsilyl cyanide $(316.7 \mu \mathrm{L}, 2.53 \mathrm{mmol}, 5$ equiv) and a $1 \mathrm{M}$ soln of $\mathrm{SnCl}_{4}$ in $\mathrm{CH}_{2} \mathrm{Cl}_{2}(101 \mu \mathrm{L}, 101 \mu \mathrm{mol}$, 0.2 equiv) were added to a stirred soln of $3(189 \mathrm{mg}$, $506 \mu \mathrm{mol})$ in dry $\mathrm{CH}_{2} \mathrm{Cl}_{2}(7.5 \mathrm{~mL})$ under argon. After $18 \mathrm{~h}$ the reaction soln was poured on a satd aq $\mathrm{NaHCO}_{3}$ soln $(23 \mathrm{~mL})$. The aq layer was extracted with $\mathrm{CH}_{2} \mathrm{Cl}_{2}$ $(3 \times 30 \mathrm{~mL})$. The combined extracts were concentrated and the dark brown residue was subjected to column chromatography to yield $\mathbf{5}(37.8 \mathrm{mg}, 110.8 \mu \mathrm{mol}, 22 \%)$ as colorless crystals, $6(57 \mathrm{mg}, 168 \mu \mathrm{mol}, 33 \%)$ as pale yellow needles and starting material $\mathbf{3}(23 \mathrm{mg}$, $61.7 \mu \mathrm{mol}, 12 \%)$.

$R_{\mathrm{f}} 0.34$ (3:1 petroleum ether-EtOAc). ${ }^{1} \mathrm{H}$ NMR $\left(\mathrm{CDCl}_{3}, 250 \mathrm{MHz}\right): \delta 5.81\left(\mathrm{~d}, 1 \mathrm{H}, J_{1,2} 5.6 \mathrm{~Hz}, \mathrm{H}-1\right)$, $5.35\left(\mathrm{~m}, 1 \mathrm{H}, J_{3,4} \approx J_{4,5} 6.4 \mathrm{~Hz}, \mathrm{H}-4\right), 4.69(\mathrm{ddd}, 1 \mathrm{H}$, $\left.J_{2,3} 2.72 \mathrm{~Hz}, J_{2,4} 1.0 \mathrm{~Hz}, \mathrm{H}-2\right), 4.26\left(\mathrm{dd}, 1 \mathrm{H}, J_{5,6}\right.$ $\left.3.4 \mathrm{~Hz}, J_{6.6^{\prime}} 12.1 \mathrm{~Hz}, \mathrm{H}-6\right), 4.18\left(\mathrm{dd}, 1 \mathrm{H}, J_{5.6^{\prime}} 5.2 \mathrm{~Hz}\right.$, H-6'), 3.97 (ddd, 1H, H-5), 3.50 (dd, 1H, H-3), 2.16 (s, $\left.3 \mathrm{H}, \mathrm{CH}_{3} \mathrm{CO}\right), 2.09\left(\mathrm{~s}, 3 \mathrm{H}, \mathrm{CH}_{3} \mathrm{CO}\right), 1.96(\mathrm{~s}, 3 \mathrm{H}$, dioxolane $\left.\mathrm{CH}_{3}\right) ;{ }^{13} \mathrm{C}$ NMR $\left(\mathrm{CDCl}_{3}, 150 \mathrm{MHz}\right): \delta 170.35$ (CO), $169.93(\mathrm{CO}), 116.39(\mathrm{CN}), 99.08$ (dioxolane $C$ ), 98.48 (C-1), 74.13 (C-2), 70.25 (C-5), 65.50 (C-4), 63.48 (C-6), 55.03 (C-3), 24.01 (dioxolane $\mathrm{CH}_{3}$ ), 20.61 $\left(\mathrm{CH}_{3} \mathrm{CO}\right), 20.60\left(\mathrm{CH}_{3} \mathrm{CO}\right)$. MALDIMS (positive mode, CHCA, MeCN) $m / z$ calcd for $\mathrm{C}_{13} \mathrm{H}_{16} \mathrm{~N}_{4} \mathrm{O}_{7}: 363.1$ $[\mathrm{M}+\mathrm{Na}]^{+}$. Found: 363.1. Anal. Calcd: C, 45.88; H, 4.74; N, 16.46. Found: C, 45.62; H, 4.55; N, 16.31 .

\subsection{Tetrazole 6}

Trimethylsilyl cyanide ( $263 \mu \mathrm{L}, 2.10 \mathrm{mmol}, 5$ equiv) and a $1 \mathrm{M}$ soln of $\mathrm{SnCl}_{4}$ in $\mathrm{CH}_{2} \mathrm{Cl}_{2}(505 \mu \mathrm{L}, 505 \mu \mathrm{mol}$, 1.2 equiv) were added to a stirred soln of $3(157 \mathrm{mg}$, $420.5 \mu \mathrm{mol})$ in dry $\mathrm{CH}_{2} \mathrm{Cl}_{2}(4 \mathrm{~mL})$ under argon at $60{ }^{\circ} \mathrm{C}$. After $2 \mathrm{~h}$ the reaction soln was poured on a satd aq $\mathrm{NaHCO}_{3}$ soln. The aq layer was extracted with $\mathrm{CH}_{2} \mathrm{Cl}_{2}$. The combined extracts were dried $\left(\mathrm{MgSO}_{4}\right)$ concentrated and the dark brown residue was subjected to column chromatography (2:1 petroleum ether-EtOAc) to yield $6(93.0 \mathrm{mg}, 273 \mu \mathrm{mol}, 65 \%)$ as a yellow oil.

$R_{\mathrm{f}} 0.32$ (2:1 petroleum ether-EtOAc). ${ }^{1} \mathrm{H}$ NMR $\left(\mathrm{CDCl}_{3}, 250 \mathrm{MHz}\right): \delta 5.73\left(\mathrm{~d}, 1 \mathrm{H}, J_{1,2} 3.9 \mathrm{~Hz}, \mathrm{H}-1\right)$, $5.59\left(\mathrm{dd}, 1 \mathrm{H}, J_{2,3} 5.8, J_{3,4} 3.1 \mathrm{~Hz}, \mathrm{H}-3\right), 5.08(\mathrm{dd}, 1 \mathrm{H}$, $\left.J_{4,5} 10.5 \mathrm{~Hz}, \mathrm{H}-4\right), 4.89(\mathrm{dd}, 1 \mathrm{H}, \mathrm{H}-2), 4.28(\mathrm{dd}, 1 \mathrm{H}$, $\left.J_{5,6} \quad 3.9 \mathrm{~Hz}, \quad J_{6,6^{\prime}} 12.4 \mathrm{~Hz}, \quad \mathrm{H}-6\right), \quad 4.16 \quad(\mathrm{dd}, \quad 1 \mathrm{H}$, $\left.J_{5,6^{\prime}} 2.1 \mathrm{~Hz}, \mathrm{H}-6^{\prime}\right), 3.45(\mathrm{~m}, 1 \mathrm{H}, \mathrm{H}-5), 2.16(\mathrm{~s}, 3 \mathrm{H}$, $\left.\mathrm{CH}_{3} \mathrm{CO}\right), 1.98\left(\mathrm{~s}, 3 \mathrm{H}, \mathrm{CH}_{3} \mathrm{CO}\right), 1.95(\mathrm{~s}, 3 \mathrm{H}$, dioxolane $\left.\mathrm{CH}_{3}\right) ;{ }^{1} \mathrm{H}$ NMR (DMSO- $\left.d_{6} 600 \mathrm{MHz}\right): \delta=5.76(\mathrm{~d}$, $\left.1 \mathrm{H}, J_{1,2} 3.9 \mathrm{~Hz}, \mathrm{H}-1\right), 5.74\left(\mathrm{dd}, 1 \mathrm{H}, J_{2,3} 5.6 \mathrm{~Hz}, J_{3,4}\right.$ $3.1 \mathrm{~Hz}, \mathrm{H}-3), 5.43$ (dd, 1H, H-2), 5.20 (dd, 1H, H-4), 4.15 (dd, $\left.1 \mathrm{H}, J_{5,6} 4.3 \mathrm{~Hz}, J_{6,6^{\prime}} 12.5 \mathrm{~Hz}, \mathrm{H}-6\right), 4.03$ (dd, $\left.1 \mathrm{H}, J_{5,6^{\prime}} 1.5 \mathrm{~Hz}, \mathrm{H}-6^{\prime}\right), 3.34(\mathrm{~m}, 1 \mathrm{H}, \mathrm{H}-5), 2.16(\mathrm{~s}, 3 \mathrm{H}$, $\left.\mathrm{CH}_{3} \mathrm{CO}\right), 1.98\left(\mathrm{~s}, 3 \mathrm{H}, \mathrm{CH}_{3} \mathrm{CO}\right), 1.95(\mathrm{~s}, 3 \mathrm{H}$, dioxolane
$\left.\mathrm{CH}_{3}\right) ;{ }^{13} \mathrm{C} \quad \mathrm{NMR}$ (DMSO- $\left.d_{6}, 150 \mathrm{MHz}\right): \quad \delta \quad 169.97$ (CO), 169.13 (CO), 155.20 (C-tetrazole), 100.97 (dioxolane C), 97.85 (C-1), 68.32 (C-2), 65.22 (C-4), 63.68 (C-5), 61.38 (C-6), 55.52 (C-3), $20.67\left(\mathrm{CH}_{3} \mathrm{CO}\right), 20.51$ $\left(\mathrm{CH}_{3} \mathrm{CO}\right.$ ), 19.73 (dioxolane $\mathrm{CH}_{3}$ ). MALDIMS (positive mode, CHCA, dioxane) $\mathrm{m} / \mathrm{z}$ calcd for $\mathrm{C}_{13} \mathrm{H}_{16} \mathrm{~N}_{4} \mathrm{O}_{7}$ : 363.1 $[\mathrm{M}+\mathrm{Na}]^{+}$. Found: 363.1. Anal. Calcd: C, 45.88; H, 4.74; N, 16.46. Found: C, 45.83; H, 4.79; N, 16.52 .

\subsection{Tetrazole 7}

A $0.05 \mathrm{M}$ soln of $\mathrm{NaOMe}$ in $\mathrm{MeOH}(0.41 \mathrm{~mL}$, 0.05 equiv) was added to a stirred soln of compound 6 $(140.2 \mathrm{~g}, 0.412 \mathrm{mmol})$ in dry $\mathrm{MeOH}(4.1 \mathrm{~mL})$. After $14 \mathrm{~h}$ ion exchange resin Amberlite IR-120 ( $\mathrm{H}^{+}$form) was added until the soln showed a $\mathrm{pH}$ of 7 . Evaporation to dryness and subsequent chromatographic purification (1:9 petroleum ether-EtOAc) afforded compound $\mathbf{6}$ as a white wax-like solid ( $88.3 \mathrm{mg}, 0.345 \mathrm{mmol}, 84 \%$ ).

$R_{\mathrm{f}} 0.19$ (1:9 petroleum ether-EtOAc). ${ }^{1} \mathrm{H}$ NMR $\left(\mathrm{D}_{2} \mathrm{O}\right.$, $600 \mathrm{MHz}$ ): $\delta 5.85\left(\mathrm{~d}, 1 \mathrm{H}, J_{1,2} 4.0 \mathrm{~Hz}, \mathrm{H}-1\right), 5.57$ (dd, $1 \mathrm{H}$, $\left.J_{2,3} 5.5 \mathrm{~Hz}, J_{3,4} 3.0 \mathrm{~Hz}, \mathrm{H}-3\right), 5.23(\mathrm{dd}, 1 \mathrm{H}, \mathrm{H}-2), 4.30$ (dd, $\left.1 \mathrm{H}, J_{4,5} 10.2 \mathrm{~Hz}, \mathrm{H}-4\right), 3.74-3.80(\mathrm{~m}, 2 \mathrm{H}, \mathrm{H}-6, \mathrm{H}-$ $\left.6^{\prime}\right), 3.07(\mathrm{~m}, 1 \mathrm{H}, \mathrm{H}-5), 2.07$ (dioxolane $\left.\mathrm{CH}_{3}\right) ;{ }^{13} \mathrm{C}$ NMR $\left(\mathrm{D}_{2} \mathrm{O}+2\right.$ drops $\left.\mathrm{MeOH}-d_{4}, 150 \mathrm{MHz}\right): \delta 156.41$ (C-tetrazole), 102.15 (dioxolane $C$ ), 99.16 (C-1), 70.35 (C-2), 68.88 (C-5), 65.27 (C-4), 60.63 (C-6), 59.83 (C3), 19.87 (dioxolane $\mathrm{CH}_{3}$ ). Anal. Calcd for $\mathrm{C}_{9} \mathrm{H}_{12} \mathrm{~N}_{4} \mathrm{O}_{5} \cdot \mathrm{H}_{2} \mathrm{O}: \mathrm{C}, 41.46 ; \mathrm{H}, 4.83 ; \mathrm{N}, 21.49$. Found: C, 41.62; H, 5.12; N, 21.54.

\subsection{3-Azido-1,2,4,6-tetra- $O$-benzoyl-3-deoxy- $\beta$-D-allo- pyranose (10)}

Compound 2 (1.01 g, $4.92 \mathrm{mmol}$ ) was dissolved in pyridine $(8 \mathrm{~mL})$ at room temperature. The colorless soln was cooled in an ice bath and benzoyl chloride $(11.44 \mathrm{~mL}$, $98.5 \mathrm{mmol}, 5$ equiv per $\mathrm{OH}$ group) was added. After stirring for $24 \mathrm{~h}$ at room temperature, the soln was concentrated under diminished pressure. The residue was suspended in EtOAc and washed with water $(80 \mathrm{~mL})$ and satd aq $\mathrm{NaHCO}_{3}$ soln $(100 \mathrm{~mL})$. The organic phase was dried $\left(\mathrm{MgSO}_{4}\right)$ and concentrated. Chromatographic purification $(8: 1 \rightarrow 5: 1 \rightarrow 3: 1$ petroleum ether-EtOAc) afforded $\mathbf{1 0}(2.34 \mathrm{~g}, 3.78 \mathrm{mmol}, 77 \%)$ as a colorless foam. Minor amounts of the corresponding $\alpha$ and $\beta$-furanose were formed as side products. They were completely separable by column chromatography.

$R_{\mathrm{f}} 0.42$ (4:1 petroleum ether-EtOAc). ${ }^{1} \mathrm{H}$ NMR $\left(\mathrm{CDCl}_{3}, 250 \mathrm{MHz}\right): \delta 7.99-8.09(\mathrm{~m}, 8 \mathrm{H}, \mathrm{Ph}), 7,36-7,65$ $(\mathrm{m}, 12 \mathrm{H}, \mathrm{Ph}) 6.49\left(\mathrm{~d}, 1 \mathrm{H}, J_{1,2} 8.5 \mathrm{~Hz}, \mathrm{H}-1\right), 5.59(\mathrm{dd}$, $\left.1 \mathrm{H}, J_{2,3} 3.4 \mathrm{~Hz}, \mathrm{H}-2\right), 5.52\left(\mathrm{dd}, 1 \mathrm{H}, J_{3,4} 3.3 \mathrm{~Hz}, J_{4,5}\right.$ $9.7 \mathrm{~Hz}, \mathrm{H}-4), 4.82$ (t, 1H, H-3), 4.62-4.69 (m, 2H, H-5, H-6), 4.47 (dd, $\left.1 \mathrm{H}, J_{5,6^{\prime}} 5.0 \mathrm{~Hz}, J_{6,6^{\prime}} 12.9 \mathrm{~Hz}, \mathrm{H}-6^{\prime}, \mathrm{Hz}\right)$; ${ }^{13} \mathrm{C}$ NMR $\left(\mathrm{CDCl}_{3}, 150 \mathrm{MHz}\right): \delta 166.10$ (CO), 165.02 (CO), 164.98 (CO), 164.67 (CO), 133.85, 133.74, 
$133.70,133.09,130.14,130.03,129.81,129.65,128.75$, $128.64,128.60,128.58,128.51,128.47,128.40,128.36$ (24 C, C-arom.), 90.81 (C-1), 71.24 (C-5), 69.81 (C-2), 68.05 (C-4), 62.97 (C-6), 61.56 (C-3). MALDIMS (positive mode, DHB, dioxane) $m / z$ calcd for $\mathrm{C}_{34} \mathrm{H}_{27} \mathrm{~N}_{3} \mathrm{O}_{9}$ : $644.2[\mathrm{M}+\mathrm{Na}]^{+}$. Found: 644.5. ESIHRMS $\mathrm{m} / \mathrm{z}$ calcd for $\mathrm{C}_{34} \mathrm{H}_{27} \mathrm{~N}_{3} \mathrm{O}_{9}: 644.1645[\mathrm{M}+\mathrm{Na}]^{+}$. Found: 644.1663 .

\subsection{1- $O$-Acetyl-3-azido-2,4,6-tri- $O$-benzoyl-3-deoxy- $\alpha, \beta$ - D-allopyranose 11}

Compound 10 (1.54 g, $2.48 \mathrm{mmol})$ was dissolved in DMF $(10 \mathrm{~mL})$ at $50^{\circ} \mathrm{C}$ and stirred for $10 \mathrm{~min}$. Hydrazinium acetate $(274.4 \mathrm{mg}, 2.97 \mathrm{mmol}, 1.2$ equiv) suspended in DMF $(2 \mathrm{~mL})$ was added and the resulting soln was stirred for $3 \mathrm{~h}$ at $50{ }^{\circ} \mathrm{C}$. The reaction mixture was diluted with $70 \mathrm{~mL}$ EtOAc and washed with $50 \mathrm{~mL}$ portions of a satd aq $\mathrm{NaCl}$ soln until the precipitation of salt had stopped. The organic phase was dried $\left(\mathrm{MgSO}_{4}\right)$ and concentrated. After passing the residue through a short bed of silica (3:1 petroleum etherEtOAc), the solvent mixture was removed under diminished pressure and dried for 3 days under vacuum over $\mathrm{P}_{2} \mathrm{O}_{5}$. The remaining pale yellow solid was dissolved in pyridine $(2.5 \mathrm{~mL})$ and then stirred with $\mathrm{Ac}_{2} \mathrm{O}(3 \mathrm{~mL})$ for $12 \mathrm{~h}$. The soln was concentrated and coevaporated three times with toluene. The crude product was purified by column chromatography to yield 11 (470 mg, $0.84 \mathrm{mmol}, 41 \%, \alpha: \beta=1: 2.9)$ as a colorless foam.

$R_{\mathrm{f}} 0.37$ (3:1 petroleum ether-EtOAc). ${ }^{1} \mathrm{H}$ NMR $\left(\mathrm{CDCl}_{3}, 250 \mathrm{MHz}\right): \delta 7.41-8.09\left(3 \mathrm{~m}, 3 \mathrm{Ph}_{\alpha}, 3 \mathrm{Ph}_{\beta}\right), 6.48$ $\left(\mathrm{d}, J_{1,2} 3.9 \mathrm{~Hz}, \mathrm{H}-1_{\alpha}\right), 6.28\left(\mathrm{~d}, J_{1,2} 8.4 \mathrm{~Hz}, \mathrm{H}-1_{\beta}\right), 5.54$ $\left(\mathrm{t}, J_{2,3} \approx 3.8 \mathrm{~Hz}, \mathrm{H}-2_{\alpha}\right), 5.46\left(\mathrm{dd}, J_{3,4} 3.2 \mathrm{~Hz}, J_{4,5}\right.$ $\left.10.0 \mathrm{~Hz}, \mathrm{H}-4_{\beta}\right), 5.40\left(\mathrm{dd}, J_{3,4} 3.3 \mathrm{~Hz}, J_{4,5} 10.1 \mathrm{~Hz}\right.$, $\mathrm{H}-4_{\alpha}$ ), 5.33 (dd, H-2 ${ }_{\beta}, J_{2,3} 3.4 \mathrm{~Hz}$ ), $4.80\left(\mathrm{t}, \mathrm{H}-3_{\alpha}\right), 4.77$ $\left(\mathrm{t}, 3-\mathrm{H}_{\beta}\right), 4.69\left(\mathrm{~m}, \mathrm{H}-5_{\alpha}\right), 4.66\left(\mathrm{dd}, J_{5,6} 2.5 \mathrm{~Hz}\right.$, $\left.J_{6,6^{\prime}} 12.4 \mathrm{~Hz}, \mathrm{H}-6_{\alpha}\right), 4.62\left(\mathrm{dd}, J_{5,6} 2.2 \mathrm{~Hz}, J_{6,6^{\prime}} 12.3 \mathrm{~Hz}\right.$, H-6 $\left.6_{\beta}\right), 4.56\left(\mathrm{~m}, \mathrm{H}-5_{\beta}\right), 4.48$ (dd, $J_{5,6^{\prime}} 4.1 \mathrm{~Hz}, \mathrm{H}-6_{\alpha}^{\prime}$ ), $4.43\left(\mathrm{dd}, J_{5,6^{\prime}} 4.6 \mathrm{~Hz}, \mathrm{H}-6_{\beta}^{\prime}\right), 2.24\left(\mathrm{~s}, 3 \mathrm{H}, \mathrm{CH}_{3} \mathrm{CO}_{\alpha}\right)$, 2.07 (s, $\left.3 \mathrm{H}, \mathrm{CH}_{3} \mathrm{CO}_{\beta}\right) ;{ }^{13} \mathrm{C} \mathrm{NMR}\left(\mathrm{CDCl}_{3}, 150 \mathrm{MHz}\right)$ : $\delta 169.12\left(\mathrm{CO}_{\alpha}\right), 169.07\left(\mathrm{CO}_{\beta}\right), 166.11\left(\mathrm{CO}_{\beta}\right), 166.09$ $\left(\mathrm{CO}_{\alpha}\right), 164.89\left(\mathrm{CO}_{\alpha}\right), 164.87\left(2 \times \mathrm{CO}_{\beta}\right), 164.85\left(\mathrm{CO}_{\alpha}\right)$, 128.39-133.89 (36C, $\left.3 \times \mathrm{Ph}_{\alpha}, 3 \times \mathrm{Ph}_{\beta}\right), 89.99\left(\mathrm{C}-1_{\beta}\right)$, $88.46\left(\mathrm{C}-1_{\alpha}\right), 71.05\left(\mathrm{C}-5_{\beta}\right), 69.75\left(\mathrm{C}-2_{\beta}\right), 68.40\left(\mathrm{C}-2_{\alpha}\right)$, $67.75\left(\mathrm{C}-4_{\beta}\right), 67.21\left(\mathrm{C}-4_{\alpha}\right), 66.14\left(\mathrm{C}-5_{\alpha}\right), 62.84\left(\mathrm{C}-6_{\beta}\right)$, $62.54\left(\mathrm{C}-6_{\alpha}\right), \quad 61.25 \quad\left(\mathrm{C}-3_{\beta}\right), \quad 58.88 \quad\left(\mathrm{C}-3_{\alpha}\right), \quad 20.98$ $\left(\mathrm{CH}_{3} \mathrm{CO}_{\alpha}\right), 20.87 \quad\left(\mathrm{CH}_{3} \mathrm{CO}_{\beta}\right)$. MALDIMS (positive mode, DHB, dioxane) $\mathrm{m} / z$ calcd for $\mathrm{C}_{29} \mathrm{H}_{25} \mathrm{~N}_{3} \mathrm{O}_{9}$ : 582.2 $[\mathrm{M}+\mathrm{Na}]^{+}$. Found: 582.3. Anal. Calcd for $\mathrm{C}_{29} \mathrm{H}_{25} \mathrm{~N}_{3} \mathrm{O}_{9}$ : C, 62.25; H, 4.50; N, 7.51. Found: C, 62.22; H, 4.41; N, 7.65 .

\subsection{Tetrazole 14}

Trimethylsilyl cyanide ( $110.5 \mu \mathrm{L}, 883 \mu \mathrm{mol}, 10$ equiv) was added to a stirred soln of $\mathbf{1 1}(49.4 \mathrm{mg}, 88.3 \mu \mathrm{mol})$ in nitromethane $(2 \mathrm{~mL})$ at room temperature under argon. $\mathrm{BF}_{3} \cdot \mathrm{OEt}_{2}$ was added in portions of $2 \mu \mathrm{L}$ every $30 \mathrm{~min}$, until TLC indicated complete consumption of starting material. The soln was poured on a satd aq $\mathrm{NaHCO}_{3}$ soln $(20 \mathrm{~mL})$ and the aq layer was extracted with $\mathrm{CH}_{2} \mathrm{Cl}_{2}(3 \times 30 \mathrm{~mL})$. The combined extracts were concentrated and the dark brown residue was subjected to column chromatography (4:1 petroleum etherEtOAc) to yield $14(23.4 \mathrm{mg}, 44.2 \mu \mathrm{mol}, 50 \%)$ as a pale yellow syrup.

$R_{\mathrm{f}} 0.28$ (4:1 petroleum ether-EtOAc). ${ }^{1} \mathrm{H}$ NMR $\left(\mathrm{CDCl}_{3}, 250 \mathrm{MHz}\right): \delta 7.41-8.17(4 \mathrm{~m}, 15 \mathrm{H}$, arom. $), 5.94$ $\left(\mathrm{d}, 1 \mathrm{H}, J_{1,2} 3.9 \mathrm{~Hz}, \mathrm{H}-1\right), 5.79\left(\mathrm{dd}, 1 \mathrm{H}, J_{2,3} 5.8 \mathrm{~Hz}, J_{3,4}\right.$ $3.0 \mathrm{~Hz}, \mathrm{H}-3), 5.68$ (dd, 1H, $\left.J_{4,5} 10.4 \mathrm{~Hz}, \mathrm{H}-4\right), 5.17$ $(\mathrm{dd}, 1 \mathrm{H}, \mathrm{H}-2), 4.68\left(\mathrm{dd}, 1 \mathrm{H}, J_{5,6} 4.1 \mathrm{~Hz}, J_{6,6^{\prime}} 12.5 \mathrm{~Hz}\right.$, $\mathrm{H}-6), 4.44$ (dd, 1H, H-6'), $3.86(\mathrm{~m}, 1 \mathrm{H}, \mathrm{H}-5) ;{ }^{13} \mathrm{C}$ NMR $\left(\mathrm{CDCl}_{3}, 150 \mathrm{MHz}\right): \delta 165.9(\mathrm{CO}), 165.3(\mathrm{CO})$, $134.14,133.33,131.83,130.85,130.30,129.74,129.32$, 128.74, 128.62, 128.45, 128.36, 126.38 (18 C, C-arom.), 102.9 (dioxolane C), 98.6 (C-1), 69.4 (C-2), 66.4 (C-4), 64.7 (C-5), 62.0 (C-6), 56.1 (C-3). MALDIMS (positive mode, DHB, dioxane) $\mathrm{m} / z$ calcd for $\mathrm{C}_{28} \mathrm{H}_{22} \mathrm{~N}_{4} \mathrm{O}_{7}$ : $549.2[\mathrm{M}+\mathrm{Na}]^{+}$. Found: 549.2. ESIHRMS $\mathrm{m} / \mathrm{z}$ calcd for $\mathrm{C}_{28} \mathrm{H}_{22} \mathrm{~N}_{4} \mathrm{O}_{7}: 565.1112[\mathrm{M}+\mathrm{K}]^{+}$. Found: 565.1111 .

\subsection{Methyl 3-azido-3-deoxy- $\alpha, \beta$-D-allofuranoside (15) and methyl 3-azido-3-deoxy-5,6- $O$-isopropylidene- $\alpha, \beta-$ D-allofuranoside (16)}

Acetyl chloride ( $86 \mu \mathrm{L}, 1.2 \mathrm{mmol})$ was added to a stirred soln of compound $1(857 \mathrm{mg}, 3 \mathrm{mmol})$ in $11.8 \mathrm{~mL}$ of $\mathrm{MeOH}$ under nitrogen at $0{ }^{\circ} \mathrm{C}$. The ice bath was removed and the reaction mixture was stirred for $48 \mathrm{~h}$ at room temperature. The soln was neutralized with $\mathrm{Et}_{3} \mathrm{~N}$ and concentrated under diminished pressure. The pale yellow residue was subjected to column chromatography to yield 15 (367 mg, $1.69 \mathrm{mmol}, 56 \%)$ and $16(250 \mathrm{mg}$, $0.97 \mathrm{mmol}, 34 \%$ ) as colorless crystals.

Compound 15: $R_{\mathrm{f}} 0.23$ (1:4 petroleum ether-EtOAc). ${ }^{1} \mathrm{H}$ NMR $\left(\mathrm{MeOH}-d_{4}, 600 \mathrm{MHz}\right): \delta 4.82\left(\mathrm{~d}, J_{1,2} 4.4 \mathrm{~Hz}\right.$, $\left.\mathrm{H}-1_{\alpha}\right), 4.74\left(\mathrm{~s}, J_{1,2}<1 \mathrm{~Hz}, \mathrm{H}-1_{\beta}\right), 4.22\left(\mathrm{dd}, J_{2,3} 7.6 \mathrm{~Hz}\right.$, $\mathrm{H}-2_{\alpha}$ ), 4.10 (d, $\left.J_{2,3} 4.2 \mathrm{~Hz}, \mathrm{H}-2_{\beta}\right), 3.92-3.97\left(\mathrm{~m}, \mathrm{H}-3_{\alpha}\right.$, $\left.\mathrm{H}-3_{\beta}, \mathrm{H}-4_{\beta}\right), 3.90\left(\mathrm{t}, J_{3,4} \approx J_{4,5} 4.2 \mathrm{~Hz}, \mathrm{H}-4_{\alpha}\right), 3.75(\mathrm{dd}$, $\left.J_{5,6} 3.3 \mathrm{~Hz}, J_{6,6^{\prime}} 11.4 \mathrm{~Hz}, \mathrm{H}-6_{\beta}\right), 3.56-3.63\left(\mathrm{~m}, \mathrm{H}-5_{\alpha}\right.$, H-6 $\left.\sigma_{\beta}^{\prime}, \mathrm{H}-6_{\alpha}, \mathrm{H}-6_{\alpha}^{\prime}\right), 3.51$ (m, H-5 $\left.5_{\alpha}\right), 3.39\left(\mathrm{~s}, \mathrm{OMe}_{\alpha}\right)$, $3.32\left(\mathrm{OMe}_{\beta}\right) ;{ }^{13} \mathrm{C}$ NMR $\left(\mathrm{MeOH}-d_{4}, 150 \mathrm{MHz}\right): \delta$ $109.76\left(\mathrm{C}-1_{\beta}\right), 103.64\left(\mathrm{C}-1_{\alpha}\right), 83.72\left(\mathrm{C}-4_{\alpha}\right), 81.65(\mathrm{C}-$ $\left.4_{\beta}\right), 77.16\left(\mathrm{C}-2_{\beta}\right), 75.39\left(\mathrm{C}-5_{\beta}\right), 74.20\left(\mathrm{C}-2_{\alpha}\right), 73.28(\mathrm{C}-$ $\left.5_{\alpha}\right), 65.39\left(\mathrm{C}-3_{\beta}\right), 64.82\left(2 \mathrm{C}, \mathrm{C}-6_{\alpha}, \mathrm{C}-6_{\beta}\right), 61.60\left(\mathrm{C}-3_{\alpha}\right)$, $55.37\left(\mathrm{OMe}_{\beta}\right), 55.31\left(\mathrm{OMe}_{\alpha}\right)$. Anal. Calcd: C, 38.36; H, 5.98; N, 19.17. Found: C, 38.50; H, 6.05; N, 19.01.

Compound 16: $R_{\mathrm{f}} 0.27$ (1:2 petroleum ether-EtOAc). ${ }^{1} \mathrm{H}$ NMR $\left(\mathrm{CDCl}_{3}, 600 \mathrm{MHz}\right): \delta 4.80\left(\mathrm{~d}, J_{1 \alpha, 2 \alpha} 4.5 \mathrm{~Hz}\right.$, $\left.\mathrm{H}-1_{\alpha}\right), 4.75$ (s, $\left.J_{1 \beta, 2 \beta}<1 \mathrm{~Hz}, \mathrm{H}-1_{\beta}\right), 4.20$ (m, H-2 ${ }_{\alpha}$ ), 4.02-4.12 (m, H- $6_{\beta}, \mathrm{H}-2_{\beta}, \mathrm{H}-3_{\beta}, \mathrm{H}-5_{\alpha}, \mathrm{H}-6_{\alpha}$ ), 3.89-3.97 
(m, H- $\left.6_{\beta}^{\prime}, \mathrm{H}-5_{\beta}, \mathrm{H}-4_{\beta}, \mathrm{H}-3_{\alpha}, \mathrm{H}-6_{\alpha}\right), 3.84$ (dd, $J_{3 \alpha, 4 \alpha}$ $\left.3.4 \mathrm{~Hz}, J_{4 \alpha, 5 \alpha} 5.9 \mathrm{~Hz}, \mathrm{H}-4_{\alpha}\right), 3.80\left(\mathrm{~m}, \mathrm{H}-6_{\alpha}\right), 3.40(\mathrm{~s}$, $\left.\mathrm{OMe}_{\alpha}\right), 3.26\left(\mathrm{OMe}_{\beta}\right), 1.41\left(\mathrm{~s}, 1 \mathrm{CH}_{3 \beta}, 1 \mathrm{CH}_{3 \alpha}\right), 1.31$ (s, $\left.\mathrm{CH}_{3 \beta}\right), 1.30\left(\mathrm{~s}, \mathrm{CH}_{3 \alpha}\right) ;{ }^{13} \mathrm{C} \mathrm{NMR}\left(\mathrm{CDCl}_{3}, 150 \mathrm{MHz}\right)$ : $\delta 109.86$ (C-isopropylidene ${ }_{\alpha}$ ), 109.77 (C-isopropylidene $\left._{\beta}\right), 107.83\left(\mathrm{C}-1_{\beta}\right), 101.92\left(\mathrm{C}-1_{\alpha}\right), 82.58\left(\mathrm{C}-4_{\alpha}\right)$, $81.99\left(\mathrm{C}-4_{\beta}\right), 77.56\left(\mathrm{C}-5_{\beta}\right), 75.50\left(\mathrm{C}-5_{\alpha}\right), 75.48\left(\mathrm{C}-2_{\beta}\right)$, $72.38\left(\mathrm{C}-2_{\alpha}\right), 67.67\left(\mathrm{C}-6_{\beta}\right), 66.34\left(\mathrm{C}-6_{\alpha}\right), 65.31\left(\mathrm{C}-3_{\beta}\right)$, $60.95\left(\mathrm{C}-3_{\alpha}\right), 55.33\left(\mathrm{OMe}_{\alpha}\right), 54.82\left(\mathrm{OMe}_{\beta}\right), 26.33$ $\left(\mathrm{CH}_{3 \beta}\right), 26.27\left(\mathrm{CH}_{3 \alpha}\right), 25.03\left(\mathrm{CH}_{3 \beta}\right), 24.61\left(\mathrm{CH}_{3 \alpha}\right)$. Anal. Calcd: C, 46.33; H, 6.61; N, 16.21. Found: C, 46.41; H, $6.74 ; \mathrm{N}, 16.00$.

\subsection{1,2,5,6-Tetra- $O$-acetyl-3-azido-3-deoxy- $\alpha, \beta$-D-allo- furanose (17)}

$\mathrm{Ac}_{2} \mathrm{O}(5 \mathrm{~mL})$ was added to a stirred soln of compound $15(271 \mathrm{mg}, 1.24 \mathrm{mmol})$ in pyridine $(10 \mathrm{~mL})$. After $12 \mathrm{~h}$ the reaction mixture was concentrated under diminished pressure and the yellow residue was filtered through a short silica column (2:1 petroleum ether-EE). The combined product fractions were dried and subsequently dissolved in a mixture of $\mathrm{Ac}_{2} \mathrm{O}(1.29 \mathrm{~mL})$, HOAc $(4.92 \mathrm{~mL})$ and concd $\mathrm{H}_{2} \mathrm{SO}_{4}(360 \mu \mathrm{L})$. After $90 \mathrm{~min}$, the reaction mixture was poured on a satd aq $\mathrm{NaHCO}_{3}$ soln. The aq layer was extracted with $\mathrm{CH}_{2} \mathrm{Cl}_{2}(3 \times 30 \mathrm{~mL})$. The combined extracts were concentrated and the dark brown residue was subjected to column chromatography to yield 17 (386 mg, $1.03 \mathrm{mmol}, 83 \%$ ) as a colorless oil.

$R_{\mathrm{f}} 0.26$ (2:1 petroleum ether-EtOAc). ${ }^{1} \mathrm{H}$ NMR $\left(\mathrm{CDCl}_{3}, 600 \mathrm{MHz}\right): \delta 6.31\left(\mathrm{~d}, J_{1,2} 4.4 \mathrm{~Hz}, \mathrm{H}-1_{\alpha}\right), 6.12$ $\left(\mathrm{s}, J_{1,2}<1 \mathrm{~Hz}, \mathrm{H}-1_{\beta}\right), 5.31\left(\mathrm{~d}, J_{2,3} 4.9 \mathrm{~Hz}, \mathrm{H}-2_{\beta}\right), 5.09-$ $5.18\left(\mathrm{~m}, \mathrm{H}-2_{\alpha}, \mathrm{H}-5_{\beta}, \mathrm{H}-5_{\alpha}\right), 4.45\left(\mathrm{dd}, J_{5,6} 3.2 \mathrm{~Hz}, J_{6,6^{\prime}}\right.$ $\left.12.2 \mathrm{~Hz}, \mathrm{H}-6_{\beta}\right), 4.38\left(\mathrm{dd}, J_{5,6} 3.9 \mathrm{~Hz}, J_{6,6^{\prime}} 12.3 \mathrm{~Hz}\right.$, $\left.\mathrm{H}-6_{\alpha}\right), 4.26\left(\mathrm{t}, J_{3,4} \approx J_{4,5} 4.3 \mathrm{~Hz}, \mathrm{H}-4_{\alpha}\right), 4.17-4.22(\mathrm{~m}$, $\left.\mathrm{H}-4_{\beta}, \mathrm{H}-3_{\alpha}\right), 4.11-4.16$ (m, H- $\left.3_{\beta}, \mathrm{H}-6_{\alpha}^{\prime}\right), 4.08$ (dd, $\left.J_{5,6^{\prime}} 5.6 \mathrm{~Hz}, \mathrm{H}-6^{\prime} \beta\right), 2.16\left(\mathrm{~s}, 1 \mathrm{CH}_{3 \beta}, 1 \mathrm{CH}_{3 \alpha}\right), 2.12(\mathrm{~s}$, $\left.1 \mathrm{CH}_{3 \beta}, \quad 1 \mathrm{CH}_{3 \alpha}\right), 2.09\left(\mathrm{~s}, \mathrm{CH}_{3 \alpha}\right), 2.08\left(\mathrm{~s}, 1 \mathrm{CH}_{3 \beta}\right.$, $\left.1 \mathrm{CH}_{3 \alpha}\right), 2.06\left(\mathrm{~s}, \mathrm{CH}_{3 \beta}\right) .{ }^{13} \mathrm{C} \mathrm{NMR}\left(\mathrm{CDCl}_{3}, 150 \mathrm{MHz}\right)$ : $\delta 170.42\left(\mathrm{CO}_{\beta}\right), 170.27\left(\mathrm{CO}_{\alpha}\right), 169.96\left(2 \mathrm{C}, 1 \mathrm{CO}_{\alpha}\right.$, $\left.1 \mathrm{CO}_{\beta}\right), 169.63\left(\mathrm{CO}_{\alpha}\right), 169.62\left(\mathrm{CO}_{\alpha}\right), 169.39\left(\mathrm{CO}_{\beta}\right)$, $168.57\left(\mathrm{CO}_{\beta}\right), 98.03\left(\mathrm{C}-1_{\beta}\right), 93.29\left(\mathrm{C}-1_{\alpha}\right), 82.13\left(\mathrm{C}-4_{\alpha}\right)$, $79.80\left(\mathrm{C}-4_{\beta}\right), 75.74\left(\mathrm{C}-2_{\beta}\right), 71.75$ (2 C, C-5 $\left.5_{\beta}, \mathrm{C}-2_{\alpha}\right)$, $70.34\left(\mathrm{C}-5_{\alpha}\right), \quad 62.24 \quad\left(\mathrm{C}-6_{\beta}\right), 61.97 \quad\left(\mathrm{C}-6_{\alpha}\right), 61.53$ $\left(\mathrm{C}-3_{\beta}\right), 58.24\left(\mathrm{C}-3_{\alpha}\right), 20.94,20.77,20.56,20.15(4 \mathrm{C}$, $\mathrm{CH}_{3} \mathrm{CO}_{\alpha}$ ), 20.89, 20.78, 20.62, 20.43 (4 C, $\mathrm{CH}_{3} \mathrm{CO}_{\beta}$ ). MALDI-HRMS (positive mode, DHB, dioxane) $\mathrm{m} / \mathrm{z}$ calcd for $\mathrm{C}_{14} \mathrm{H}_{19} \mathrm{~N}_{3} \mathrm{O}_{9}: 396.1091[\mathrm{M}+\mathrm{Na}]^{+}$. Found: 396.0990 .

\subsection{2,5,6-Tri- $O$-acetyl-3-azido-3-deoxy- $\beta$-D-allofuran- osyl cyanide (18) and tetrazole 19}

Trimethylsilyl cyanide (136 $\mu \mathrm{L}, 1.09 \mathrm{mmol}, 5$ equiv) and a $1 \mathrm{M}$ soln of $\mathrm{SnCl}_{4}$ in $\mathrm{CH}_{2} \mathrm{Cl}_{2}(239 \mu \mathrm{L}, 239 \mu \mathrm{mol}$, 1.1 equiv) were added to a stirred soln of compound
$17(81 \mathrm{mg}, 217 \mu \mathrm{mol})$ in dry $\mathrm{CH}_{2} \mathrm{Cl}_{2}(4 \mathrm{~mL})$ under argon. The mixture was heated in an oil bath $\left(60^{\circ} \mathrm{C}\right)$. After $90 \mathrm{~min}$ the reaction soln was poured on a satd aq $\mathrm{NaHCO}_{3}$ soln $(30 \mathrm{~mL})$. The aq layer was extracted with $\mathrm{CH}_{2} \mathrm{Cl}_{2}(3 \times 50 \mathrm{~mL})$. The combined extracts were concentrated and the dark brown residue was subjected to column chromatography to yield 18 (20 mg, $58.8 \mu \mathrm{mol}, 27 \%$ ) as a colorless oil and 19 (38 mg, $112 \mu \mathrm{mol}, 52 \%$ ) as a pale yellow oil.

Compound 18: $R_{\mathrm{f}} 0.44$ (3:2 petroleum ether-EtOAc). ${ }^{1} \mathrm{H}$ NMR $\left(\mathrm{CDCl}_{3}, 250 \mathrm{MHz}\right): \delta 5.55\left(\mathrm{dd}, 1 \mathrm{H}, J_{1,2}\right.$ $\left.3.2 \mathrm{~Hz}, J_{2,3} 5.2 \mathrm{~Hz}, \mathrm{H}-2\right), 5.34$ (dd, 1H, H-5), 4.67 (d, $1 \mathrm{H}, \mathrm{H}-1), 4.35-4.43$ (m, 2H, H-3, H-6), 4.12-4.21 (m, $\left.2 \mathrm{H}, \mathrm{H}-4, \mathrm{H}-6^{\prime}\right), 2.21$ (s, 3H, $\left.\mathrm{CH}_{3} \mathrm{CO}\right), 2.17$ (s, 3H, $\left.\mathrm{CH}_{3} \mathrm{CO}\right), 2.10\left(\mathrm{~s}, 3 \mathrm{H}, \mathrm{CH}_{3} \mathrm{CO}\right) ;{ }^{13} \mathrm{C} \mathrm{NMR}\left(\mathrm{CDCl}_{3}\right.$, $150 \mathrm{MHz}$ ): $\delta 170.329$ (CO), 169.76 (CO), 169.38 (CO), 115.41 (CN), 80.95 (C-4), 75.82 (C-2), 69.71 (C-5), 69.32 (C-1), 61.59 (C-6), 60.75 (C-3), 20.91 ( $\left.\mathrm{CH}_{3} \mathrm{CO}\right)$, $20.61\left(\mathrm{CH}_{3} \mathrm{CO}\right), 20.35\left(\mathrm{CH}_{3} \mathrm{CO}\right)$. MALDIMS (positive mode, $\mathrm{CHCA}, \mathrm{MeCN}) \mathrm{m} / z$ calcd for $\mathrm{C}_{13} \mathrm{H}_{16} \mathrm{~N}_{4} \mathrm{O}_{7}$ : 363.1 [M+Na $]^{+}$. Found: 363.1. Anal. Calcd: C, 45.88; H, 4.74; N, 16.46. Found: C, 45.78; H, 4.80; N, 16.51.

Compound 19: $R_{\mathrm{f}} 0.28$ (1:1 petroleum ether-EtOAc). ${ }^{1} \mathrm{H} \mathrm{NMR}\left(\mathrm{CDCl}_{3}, 600 \mathrm{MHz}\right): \delta 5.84\left(\mathrm{~d}, 1 \mathrm{H}, J_{1,2} 3.9 \mathrm{~Hz}\right.$, $\mathrm{H}-1), 5.62\left(\mathrm{dd}, 1 \mathrm{H}, J_{2,3} 5.5 \mathrm{~Hz}, \mathrm{H}-2\right), 5.41$ (d, $1 \mathrm{H}$, $\left.J_{3,4}<1 \mathrm{~Hz}, \mathrm{H}-3\right), 5.02$ (m, 1H, H-5), 4.55 (d, 1H, $J_{4,5}$ $6.5 \mathrm{~Hz}, \mathrm{H}-4), 4.44$ (dd, $1 \mathrm{H}, J_{5,6} 3.3 \mathrm{~Hz}, J_{6,6^{\prime}} 12.5 \mathrm{~Hz}$, $\mathrm{H}-6), 4.20\left(\mathrm{dd}, 1 \mathrm{H}, J_{5,6^{\prime}} 4.6 \mathrm{~Hz}, \mathrm{H}-6^{\prime}\right), 2.19$ (s, 3H, $\mathrm{CH}_{3} \mathrm{CO}$ ), 2.12 (s, 3H, $\left.\mathrm{CH}_{3} \mathrm{CO}\right), 2.05$ (s, 3H, dioxolane $\left.\mathrm{CH}_{3}\right) ;{ }^{13} \mathrm{C} \mathrm{NMR}\left(\mathrm{CDCl}_{3}, 150 \mathrm{MHz}\right): \delta 170.24(\mathrm{CO})$, 169.79 (CO), 154.60 (C-tetrazole), 103.43 (C-1), 102.79 (dioxolane C), 84.78 (C-4), 77.73 (C-2), 70.27 (C-5), 61.90 (C-6), 57.414 (C-3), 20.93 (dioxolane $\mathrm{CH}_{3}$ ), 20.63 $\left(\mathrm{CH}_{3} \mathrm{CO}\right), 19.04\left(\mathrm{CH}_{3} \mathrm{CO}\right)$. MALDIMS (positive mode, CHCA, MeCN) $m / z$ calcd for $\mathrm{C}_{13} \mathrm{H}_{16} \mathrm{~N}_{4} \mathrm{O}_{7}: 363.1$ $[\mathrm{M}+\mathrm{Na}]^{+}$. Found: 363.1. Anal. Calcd: C, 45.88; H, 4.74; N, 16.46. Found: C, 45.80; H, 4.76; N, 16.48.

\subsection{5,6-Di- $O$-acetyl-3-azido-3-deoxy-1,2- $O$-(1-exo-cya- noethylidene)- $\alpha$-D-allofuranose (20) and 5,6-Di- $O$-acetyl- 3-azido-3-deoxy-1,2-O-(1-endo-cyanoethylidene)- $\alpha$-D- allofuranose (21)}

Trimethylsilyl cyanide (150 $\mu \mathrm{L}, 0.1 .20 \mathrm{mmol}, 6$ equiv) and a $1 \mathrm{M}$ soln of $\mathrm{SnCl}_{4}$ in $\mathrm{CH}_{2} \mathrm{Cl}_{2}(100 \mu \mathrm{L}, 100 \mu \mathrm{mol}$, 0.5 equiv) were added to a stirred soln of compound $17(75 \mathrm{mg}, 201 \mu \mathrm{mol})$ in dry $\mathrm{CH}_{2} \mathrm{Cl}_{2}(4 \mathrm{~mL})$ under argon. After $15 \mathrm{~h}$ the reaction soln was poured on satd aq $\mathrm{NaHCO}_{3}$ soln $(25 \mathrm{~mL})$. The aq layer was extracted with $\mathrm{CH}_{2} \mathrm{Cl}_{2}(3 \times 20 \mathrm{~mL})$. The combined extracts were concentrated and the dark brown residue was taken up in acetonitrile and filtered. Purification of the remaining soln by HPLC afforded 21 (13 mg, $38 \mu \mathrm{mol}, 19 \%$ ) as a white powder and 21 (4 mg, $12 \mu \mathrm{mol}, 6 \%$ ) as a colorless oil. In addition, cyanide $18(13 \mathrm{mg}, 38 \mu \mathrm{mol}, 19 \%)$ and tetrazole 19 (25 mg, $73.5 \mu \mathrm{mol}, 37 \%)$ were isolated. 
Compound 20: $R_{\mathrm{f}} 0.34$ (3:1 petroleum ether-EtOAc). ${ }^{1} \mathrm{H} \mathrm{NMR}\left(\mathrm{CDCl}_{3}, 250 \mathrm{MHz}\right): \delta 5.99\left(\mathrm{~d}, 1 \mathrm{H}, J_{1,2} 4.0 \mathrm{~Hz}\right.$, $\mathrm{H}-1), 5.32$ (m, 1H, H-5), 4.94 (dd, 1H, J $J_{2,3} 4.93 \mathrm{~Hz}$, $\mathrm{H}-2), 4.40$ (dd, $\left.1 \mathrm{H}, J_{5,6} 3.8 \mathrm{~Hz}, J_{6,6^{\prime}} 12.1 \mathrm{~Hz}, \mathrm{H}-6\right)$, $4.17\left(\mathrm{dd}, 1 \mathrm{H}, J_{5.6^{\prime}} 6.5 \mathrm{~Hz}, \mathrm{H}-6^{\prime}\right), 4.11\left(\mathrm{dd}, 1 \mathrm{H}, J_{3,4}\right.$ $\left.9.6 \mathrm{~Hz}, J_{4,5} 5.7 \mathrm{~Hz}, \mathrm{H}-4\right), 3.62$ (dd, 1H, H-3), 2.14 (s, 3H, $\left.\mathrm{CH}_{3} \mathrm{CO}\right), 2.08\left(\mathrm{~s}, 3 \mathrm{H}, \mathrm{CH}_{3} \mathrm{CO}\right), 1.91(\mathrm{~s}, 3 \mathrm{H}$, dioxolane $\left.\mathrm{CH}_{3}\right) ;{ }^{13} \mathrm{C}$ NMR $\left(\mathrm{CDCl}_{3}, 150 \mathrm{MHz}\right): \delta$ 170.36 (CO), 169.83 (CO), 116.38 (CN), 104.74 (C-1), 100.84 (dioxolane C), 81.11 (C-2), 76.11 (C-4), 69.97 (C-5), 62.17 (C-6), 61.47 (C-3), 24.31 (dioxolane $\left.\mathrm{CH}_{3}\right), 20.71\left(\mathrm{CH}_{3} \mathrm{CO}\right), 20.59\left(\mathrm{CH}_{3} \mathrm{CO}\right)$. MALDIMS (positive mode, CHCA, MeCN) $\mathrm{m} / \mathrm{z}$ calcd for $\mathrm{C}_{13} \mathrm{H}_{16} \mathrm{~N}_{4} \mathrm{O}_{7}$ : $363.1[\mathrm{M}+\mathrm{Na}]^{+}$. Found: 363.1. Anal. Calcd: C, 45.88; H, 4.74; N, 16.46. Found: C, 45.62; $\mathrm{H}, 4.55 ; \mathrm{N}, 16.31$.

Compound 21: $R_{\mathrm{f}} 0.34$ (3:1 petroleum ether-EtOAc). ${ }^{1} \mathrm{H}$ NMR $\left(\mathrm{CDCl}_{3}, 600 \mathrm{MHz}\right): \delta 6.14\left(\mathrm{~d}, 1 \mathrm{H}, J_{1,2}\right.$ $3.8 \mathrm{~Hz}, \mathrm{H}-1), 5.29$ (m, 1H, H-5), 5.18 (t, 1H, H-2), 4.41 (dd, $\left.1 \mathrm{H}, J_{5,6} 3.5 \mathrm{~Hz}, J_{6,6^{\prime}} 12.2 \mathrm{~Hz}, \mathrm{H}-6\right), 4.19$ (dd, $1 \mathrm{H}$, $\left.J_{5,6^{\prime}} 6.1 \mathrm{~Hz}, \mathrm{H}-6^{\prime}\right), 4.13\left(\mathrm{dd}, 1 \mathrm{H}, J_{3,4} 9.5 \mathrm{~Hz}, J_{4,5} 6.1 \mathrm{~Hz}\right.$, $\mathrm{H}-4), 3.47$ (dd, 1H, J $2,3.1 \mathrm{~Hz}, \mathrm{H}-3), 2.14$ (s, 3H, $\mathrm{CH}_{3} \mathrm{CO}$ ), 2.09 (s, 3H, $\mathrm{CH}_{3} \mathrm{CO}$ ), 1.87 (s, 3H, dioxolane $\left.\mathrm{CH}_{3}\right) ;{ }^{13} \mathrm{C} \mathrm{NMR}\left(\mathrm{CDCl}_{3}, 150 \mathrm{MHz}\right): \delta 170.44$ (CO), $169.93(\mathrm{CO}), 115.59(\mathrm{CN}), 106.11$ (C-1), 104.67 (dioxolane C), 83.60 (C-2), 76.40 (C-4), 70.18 (C-5), 62.18 (C-6), 61.86 (C-3), $20.77\left(\mathrm{CH}_{3}\right), 20.66\left(\mathrm{CH}_{3}\right), 20.63$ $\left(\mathrm{CH}_{3}\right)$. MALDIMS (positive mode, CHCA, MeCN) $\mathrm{m} / \mathrm{z}$ calcd for $\mathrm{C}_{13} \mathrm{H}_{16} \mathrm{~N}_{4} \mathrm{O}_{7}: 363.1[\mathrm{M}+\mathrm{Na}]^{+}$. Found: 363.1 .

\subsection{Methyl 3-azido-2,5,6-tri- $O$-benzoyl-3-deoxy- $\alpha, \beta$-D- allofuranoside (22)}

Compound 15 (948 mg, $4.32 \mathrm{mmol}$ ) was dissolved in pyridine $(10 \mathrm{~mL})$ at room temperature. The colorless soln was cooled in an ice bath and benzoyl chloride ( $2.26 \mathrm{~mL}, 19.44 \mathrm{mmol}, 4.5$ equiv per $\mathrm{OH}$ group) was added. After stirring for $48 \mathrm{~h}$ at room temperature the soln was concentrated under diminished pressure. The residue was suspended in EtOAc $(100 \mathrm{~mL})$ and washed with water $(100 \mathrm{~mL})$ and sat. $\mathrm{NaCl}$ soln $(100 \mathrm{~mL})$. The organic phase was dried $\left(\mathrm{MgSO}_{4}\right)$ and concentrated. Chromatographic purification $(8: 1 \rightarrow 5: 1$ petroleum ether-EtOAc) afforded 22 (2.05 g, $3.86 \mathrm{mmol}, 89 \%)$ as a colorless foam.

Compound $\beta-22: \quad R_{\mathrm{f}} 0.38 \quad(4: 1$ petroleum etherEtOAc). IR (thin film): $v 2113\left(\mathrm{~N}_{3}\right), 1725 \mathrm{~cm}^{-1}$ (CO). ${ }^{1} \mathrm{H} \mathrm{NMR}\left(\mathrm{CDCl}_{3}, 250 \mathrm{MHz}\right): \delta 7.99-8.09\left(\mathrm{~m}, \mathrm{Ph}_{\beta}\right)$, 7.36-7.65 (m, $\left.\mathrm{Ph}_{\beta}\right), \quad 5.75\left(\mathrm{~m}, \mathrm{H}-5_{\beta}\right), \quad 5.54 \quad(\mathrm{~d}$, $\left.J_{1,2}<1 \mathrm{~Hz}, J_{2,3} 4.7 \mathrm{~Hz}, \mathrm{H}-2_{\beta}\right), 5.07$ (s, H-1 $\left.{ }_{\beta}\right), 4.88$ (dd, $\left.J_{5,6} 3.1 \mathrm{~Hz}, J_{6,6^{\prime}} 12.1 \mathrm{~Hz}, \mathrm{H}-6_{\beta}\right), 4.68\left(\mathrm{dd}, J_{5,6^{\prime}} 6.3 \mathrm{~Hz}\right.$, H- $\left.6_{\beta}^{\prime}\right), 4.57\left(\mathrm{t}, J_{3,4} \approx J_{4,5} \approx 7.6 \mathrm{~Hz}, \mathrm{H}-4_{\beta}\right), 4.51\left(\mathrm{dd}, J_{2,3}\right.$ $\left.4.7 \mathrm{~Hz}, \mathrm{H}-3_{\beta}\right), 4.47\left(\mathrm{~s}, \mathrm{OMe}_{\beta}\right) .{ }^{13} \mathrm{C} \mathrm{NMR}\left(\mathrm{CDCl}_{3}\right.$, $150 \mathrm{MHz}): \delta 165.74\left(\mathrm{CO}_{\beta}\right), 165.37 \quad\left(\mathrm{CO}_{\beta}\right), 164.98$ $\left(\mathrm{CO}_{\beta}\right), 128.36-134.26$ (12 C, C-arom.), $106.11\left(\mathrm{C}-1_{\beta}\right)$, $78.76\left(\mathrm{C}-4_{\beta}\right), 76.61\left(\mathrm{C}-2_{\beta}\right), 72.33\left(\mathrm{C}-5_{\beta}\right), 62.93\left(\mathrm{C}-6_{\beta}\right)$,
$61.75\left(\mathrm{C}-3_{\beta}\right), 55.19\left(\mathrm{OMe}_{\beta}\right)$. MALDIMS (positive mode, CHCA, dioxane) $\mathrm{m} / \mathrm{z}$ calcd for $\mathrm{C}_{28} \mathrm{H}_{25} \mathrm{~N}_{3} \mathrm{O}_{8}: 554.2$ $[\mathrm{M}+\mathrm{Na}]^{+}$. Found: 554.4. Anal. Calcd: C, 63.27; H, 4.74; N, 7.91. Found: C, 63.13; H, 4.86; N, 7.76.

Compound $\alpha-22: \quad R_{\mathrm{f}} 0.38 \quad(4: 1$ petroleum etherEtOAc). ${ }^{1} \mathrm{H}$ NMR $\left(\mathrm{CDCl}_{3}, 250 \mathrm{MHz}\right): \delta$ 7.99-8.09 (m, $\left.\mathrm{Ph}_{\alpha}\right), 7.38-7.56\left(\mathrm{~m}, \mathrm{Ph}_{\alpha}\right), 5.75\left(\mathrm{~m}, \mathrm{H}-5_{\alpha}\right), 5.29\left(\mathrm{~d}, J_{1,2}\right.$ $\left.4.2 \mathrm{~Hz}, \mathrm{H}-1_{\alpha}\right), 5.25\left(\mathrm{dd}, J_{2,3} 4.2, \mathrm{H}-2_{\alpha}\right), 4.82\left(\mathrm{dd}, J_{5,6}\right.$ $\left.3.4 \mathrm{~Hz}, J_{6,6^{\prime}} 12.2, \mathrm{H}-6_{\alpha}\right), 4.69\left(\mathrm{dd}, J_{5,6^{\prime}} 6.2 \mathrm{~Hz}, \mathrm{H}-6_{\alpha}^{\prime}\right)$, $4.55\left(\mathrm{dd}, J_{3,4} 7.2 \mathrm{~Hz}, \mathrm{H}-3_{\alpha}\right), 4.46\left(\mathrm{t}, \mathrm{H}-4_{\alpha}\right), 3.44(\mathrm{~s}$, $\left.\mathrm{OMe}_{\alpha}\right) \cdot{ }^{13} \mathrm{C} \mathrm{NMR}\left(\mathrm{CDCl}_{3}, 150 \mathrm{MHz}\right): \delta 128.36$ 133.37 (12 C, C-arom.), $100.93\left(\mathrm{C}-1_{\alpha}\right), 79.71\left(\mathrm{C}-4_{\alpha}\right)$, $73.02\left(\mathrm{C}-2_{\alpha}\right), 71.22\left(\mathrm{C}-5_{\alpha}\right), 62.70\left(\mathrm{C}-6_{\alpha}\right), 58.87\left(\mathrm{C}-3_{\alpha}\right)$, $55.19\left(\mathrm{OMe}_{\alpha}\right)$. MALDIMS (positive mode, CHCA, dioxane) $m / z$ calcd for $\mathrm{C}_{28} \mathrm{H}_{25} \mathrm{~N}_{3} \mathrm{O}_{8}: 554.2[\mathrm{M}+\mathrm{Na}]^{+}$. Found: 554.4 .

\subsection{1- $O$-Acetyl-3-azido-2,5,6-tri- $O$-benzoyl-3-deoxy- $\alpha, \beta$-D-allofuranose (23)}

Compound $22(2.01 \mathrm{~g}, 3.78 \mathrm{mmol})$ was dissolved in $\mathrm{Ac}_{2} \mathrm{O}(3.55 \mathrm{~mL}), \mathrm{HOAc}(15.12 \mathrm{~mL})$, and concd $\mathrm{H}_{2} \mathrm{SO}_{4}$ $(990 \mu \mathrm{L})$. After $35 \mathrm{~min}$ the reaction mixture was poured on a satd aq $\mathrm{NaHCO}_{3}$ soln. The aq layer was extracted with $\mathrm{CH}_{2} \mathrm{Cl}_{2}(3 \times 50 \mathrm{~mL})$. The combined extracts were dried $\left(\mathrm{MgSO}_{4}\right)$, concentrated, and the dark brown residue was subjected to column chromatography to yield $23(1.90 \mathrm{~g}, 3.40 \mathrm{mmol}, 90 \%)$ as a colorless oil.

Compound $\beta-23: \quad R_{\mathrm{f}} 0.38 \quad(4: 1$ petroleum etherEtOAc). ${ }^{1} \mathrm{H} \mathrm{NMR}\left(\mathrm{CDCl}_{3}, 250 \mathrm{MHz}\right): \delta 8.07-8.11(\mathrm{~m}$, $4 \mathrm{H}$, arom.), 8.00-8.03 (m, 2H, arom.), 7.54-7.63 (m, $3 \mathrm{H}$, arom.), 7.41-7.50 (m, 6H, arom.), $6.34(\mathrm{~s}, 1 \mathrm{H}$, $\left.J_{1,2}<1 \mathrm{~Hz}, \mathrm{H}-1\right), 5.76$ (m, 1H, H-5), 5.62 (d, 1H, $J_{2,3}$ $4.3 \mathrm{~Hz}, \mathrm{H}-2), 4.79\left(\mathrm{dd}, 1 \mathrm{H}, J_{5,6} 3.6 \mathrm{~Hz}, J_{6,6^{\prime}} 12.1 \mathrm{~Hz}\right.$, H-6), $4.60\left(\mathrm{dd}, 1 \mathrm{H}, J_{5.6^{\prime}} 6.4 \mathrm{~Hz}, \mathrm{H}-6^{\prime}\right), 4.50-4.56(\mathrm{~m}$, $2 \mathrm{H}, \mathrm{H}-4, \mathrm{H}-3), 1.92$ (s, 3H, $\left.\mathrm{CH}_{3} \mathrm{CO}\right) .{ }^{13} \mathrm{C} \mathrm{NMR}\left(\mathrm{CDCl}_{3}\right.$, $150 \mathrm{MHz}$ ): $\delta 168.70$ (CO), 168.03 (CO), 165.51 (CO), 165.18 (CO), 133.87, 133.64, 133.27, 130.03, 129.89, $129.69,129.45,129.15,128.61,128.58,128.50,128.47$ (18 C, C-arom.), 98.21 (C-1), 80.36 (C-4), 76.39 (C-2), 71.81 (C-5), 62.92 (C-6), 61.36 (C-3), $20.67\left(\mathrm{CH}_{3}\right)$. MALDIMS (positive mode, CHCA, dioxane) $\mathrm{m} / \mathrm{z}$ calcd for $\mathrm{C}_{29} \mathrm{H}_{25} \mathrm{~N}_{3} \mathrm{O}_{9}$ : $582.2[\mathrm{M}+\mathrm{Na}]^{+}$. Found: 582.4. Anal. Calcd: C, 62.25; H, 4.50; N, 7.51. Found: C, 62.13; H, 4.70; N, 7.56.

Compound $\alpha-23: \quad R_{\mathrm{f}} 0.38 \quad(4: 1$ petroleum etherEtOAc). ${ }^{1} \mathrm{H}$ NMR $\left(\mathrm{CDCl}_{3}, 250 \mathrm{MHz}\right): \delta 8.01-8.09(\mathrm{~m}$, 6H, arom.), 7.55-7.64 (m, 3H, arom.), 7.43-7.50 (m, $6 \mathrm{H}$, arom.), 6.57 (s, $\left.1 \mathrm{H} J_{1,2} 4.4 \mathrm{~Hz}, \mathrm{H}-1\right), 5.68(\mathrm{~m}, 1 \mathrm{H}$, H-5), 5.45 (dd, 1H, $\left.J_{2,3} 7.6 \mathrm{~Hz}, \mathrm{H}-2\right), 4.76$ (dd, $1 \mathrm{H}, J_{5,6}$ $\left.3.9 \mathrm{~Hz}, J_{6,6^{\prime}} 12.3 \mathrm{~Hz}, \mathrm{H}-6\right), 4.63\left(\mathrm{dd}, 1 \mathrm{H}, J_{5,6^{\prime}} 5.5 \mathrm{~Hz}\right.$, H-6 $), 4.58\left(\mathrm{t}, 1 \mathrm{H}, J_{4,5} \approx 4.4 \mathrm{~Hz}, \mathrm{H}-4\right), 4.54(\mathrm{dd}, 1 \mathrm{H}$, $\left.J_{3,4} 4.0 \mathrm{~Hz}, \mathrm{H}-3\right), 2.14$ (s, 3H, CH $\left.3 \mathrm{CO}\right) .{ }^{13} \mathrm{C} \mathrm{NMR}$ $\left(\mathrm{CDCl}_{3}, 150 \mathrm{MHz}\right): \delta 169.39(\mathrm{CO}), 165.98(\mathrm{CO}), 165.35$ (CO), 165.32 (CO), 133.88, 133.70, 133.36, 129.95, $129.83,129.71,129.34,129.02,128.68,128.55,128.43$ 
(18 C, C-arom.), 93.63 (C-1), 82.50 (C-4), 72.49 (C-2), 71.19 (C-5), 62.78 (C-6), 58.68 (C-3), $21.04\left(\mathrm{CH}_{3}\right)$. MALDIMS (positive mode, CHCA, dioxane) $\mathrm{m} / \mathrm{z}$ calcd for $\mathrm{C}_{29} \mathrm{H}_{25} \mathrm{~N}_{3} \mathrm{O}_{9}$ : $582.2[\mathrm{M}+\mathrm{Na}]^{+}$. Found: 582.4.

\subsection{3-Azido-2,5,6-tri- $O$-benzoyl-3-deoxy- $\beta$-D-allofuran- osyl cyanide (24)}

Trimethylsilyl cyanide ( $369 \mu \mathrm{L}, 2.95 \mathrm{mmol}, 5$ equiv) and a $1 \mathrm{M}$ soln of $\mathrm{SnCl}_{4}$ in $\mathrm{CH}_{2} \mathrm{Cl}_{2}(650 \mu \mathrm{L}, 650 \mu \mathrm{mol}$, 1.1 equiv) were added to a stirred soln of $23(331 \mathrm{mg}$, $591 \mu \mathrm{mol})$ in dry $\mathrm{CH}_{2} \mathrm{Cl}_{2}(12 \mathrm{~mL})$ under argon. The mixture was heated in an oil bath $\left(60^{\circ} \mathrm{C}\right)$. After $4 \mathrm{~h}$ the reaction soln was poured on a satd aq $\mathrm{NaHCO}_{3}$ solution $(60 \mathrm{~mL})$. The aq layer was extracted with $\mathrm{CH}_{2} \mathrm{Cl}_{2}$ $(3 \times 50 \mathrm{~mL})$. The combined extracts were dried $\left(\mathrm{MgSO}_{4}\right)$, concentrated, and the dark brown residue was subjected to column chromatography to yield $\mathbf{2 4}$ (202 mg, $384 \mu \mathrm{mol}, 65 \%$ ) as a colorless oil.

$R_{\mathrm{f}} 0.33$ (5:1 petroleum ether-EtOAc). IR (thin film): $v 2116\left(\mathrm{~N}_{3}\right), 1728 \mathrm{~cm}^{-1}(\mathrm{CO}) .{ }^{1} \mathrm{H} \mathrm{NMR}\left(\mathrm{CDCl}_{3}\right.$, $250 \mathrm{MHz}): \delta 8.02-8.11(\mathrm{~m}, 6 \mathrm{H}$, arom.), 7.43-7.51 (m, $9 \mathrm{H}$, arom.), 5.83 (dd, $J_{1,2} 3.8 \mathrm{~Hz}, J_{2,3} 5.2 \mathrm{~Hz}, \mathrm{H}-2$ ), 5.76 (m, H-5), 4.86 (d, H-1), 4.78 (dd, J $J_{5,6} 3.8 \mathrm{~Hz}$, $\left.J_{6.6^{\prime}} 12.3 \mathrm{~Hz}, \mathrm{H}-6\right), 4.65-4.69$ (m, H-6', H-3), 4.45 $\left(\mathrm{t}, \quad J_{3,4} \approx J_{4,5} \quad 6.4 \mathrm{~Hz}, \quad \mathrm{H}-4\right) .{ }^{13} \mathrm{C} \quad \mathrm{NMR} \quad\left(\mathrm{CDCl}_{3}\right.$, $150 \mathrm{MHz}): \delta 165.96$ (CO), $165.49(\mathrm{CO}), 165.15(\mathrm{CO})$, $134.27,133.75,133.38,130.08,129.90,129.69$, 129.28, $128.85,128.75,128.60,128.52,127.79$ (18 C, C-arom.), $115.28(\mathrm{CN}), 80.91(\mathrm{C}-4), 76.10(\mathrm{C}-2), 71.00$ (C-5), 69.35 (C-1), 62.59 (C-6), 61.96 (C-3). MALDIMS (positive mode, DHB, dioxane) $m / z$ calcd for $\mathrm{C}_{28} \mathrm{H}_{22} \mathrm{~N}_{4} \mathrm{O}_{7}$ : 549.1 $[\mathrm{M}+\mathrm{Na}]^{+}$. Found: 549.2. ESIHRMS $\mathrm{m} / \mathrm{z}$ calcd 549.1386 [M+Na $]^{+}$. Found: 549.1367. Anal. Calcd: C, 63.87; H, 4.21; N, 10.64. Found: C, 63.20; H, 4.49; N, 10.04 .

\section{Acknowledgment}

Financial support by the Deutsche Forschungsgemeinschaft is gratefully acknowledged.

\section{References}

1. Master, H. E.; Khan, S. I.; Poojari, K. A. Bioorg. Med. Chem. 2005, 13, 4891-4899.

2. Herr, R. J. Bioorg. Med. Chem. 2002, 10, 3379-3393.

3. Kimura, T.; Hamada, Y.; Stochaj, M.; Ikari, H.; Nagamine, A.; Abdel-Rahman, H.; Igawa, N.; Hidaka, K.; Nguyen, J.-T.; Saito, K.; Hayashi, Y.; Kiso, Y. Bioorg. Med. Chem. Lett. 2006, 16, 2380-2386.

4. Hamada, Y.; Igawa, N.; Ikari, H.; Ziora, Z.; Nguyen, J.-T.; Yamani, A.; Hidaka, K.; Kimura, T.; Saito, K.; Hayashi, Y.; Ebina, M.; Ishiura, S.; Kiso, Y. Bioorg. Med. Chem. 2006, 16, 4354-4359.
5. Wu, S.; Fluxe, A.; Sheffer, J.; Janusz, J. M.; Blass, B. E.; White, R.; Jackson, C.; Hedges, R.; Murawsky, M.; Fang, B.; Fadayel, G. M.; Hare, M.; Djandjighian, L. Bioorg. Med. Chem. 2006, 16, 6213-6218.

6. Burgos-Lepley, C. E.; Thompson, L. R.; Kneen, C. O.; Osborne, S. A.; Bryans, J. S.; Capiris, T.; Suman-Chauhan, N.; Dooley, D. J.; Donovan, C. M.; Field, M. J.; Vartanian, M. G.; Kinsora, J. J.; Lotarski, S. M.; El-Kattan, A.; Walters, K.; Cherukury, M.; Taylor, C. P.; Wustrow, D. J.; Schwarz, J. B. Bioorg. Med. Chem. 2006, 16, 2333-2336.

7. Schwarz, J. B.; Colbry, N. L.; Zhu, Z.; Nichelson, B.; Barta, N. S.; Lin, K.; Hudack, R. A.; Gibbons, S. E.; Galatsis, P.; DeOrazio, R. J.; Manning, D. D.; Vartanian, M. G.; Kinsora, J. J.; Lotarski, S. M.; Li, Z.; R., D. M.; ElKattan, A.; Thorpe, A. J.; Donevan, S. D.; Taylor, C. P.; Wustrow, D. J. Bioorg. Med. Chem. 2006, 16, 3559-3563.

8. Carpenter, W. R. J. Org. Chem. 1962, 27, 2085-2088.

9. Quast, H.; Bieber, L. Tetrahedron Lett. 1976, 17, 14851486.

10. Krayushkin, M. M.; Beskopyl'nyi, A. M.; Zlotin, S. G.; Luk'yanov, O. A.; Zhulin, V. M. Izv. Akad. Nauk SSSR, Ser. Khim. 1980, 2668.

11. Demko, Z. P.; Sharpless, K. B. Angew. Chem., Int. Ed. 2002, 41, 2110-2113.

12. Demko, Z. P.; Sharpless, K. B. Angew. Chem., Int. Ed. 2002, 41, 2113-2116.

13. Smith, P. A. S.; Clegg, J. M.; Hall, J. H. J. Org. Chem. 1958, 23, 524-529.

14. Fusco, R.; Garanti, L.; Zecchi, G. J. Org. Chem. 1975, 40, 1906-1909.

15. Garanti, L.; Zecchi, G. J. Org. Chem. 1980, 45, 4767-4769.

16. Korakas, D.; Kimbaris, A.; Varvounis, G. Tetrahedron 1996, 52, 10751-10760.

17. Porter, T. C.; Smalley, R. K.; Teguiche, M.; Purwono, B. Synthesis 1997, 773-777.

18. Davis, B. G.; Brandstetter, T. W.; Hackett, L.; Winchester, B. G.; Nash, R. J.; Watson, A. A.; Griffiths, R. C.; Smith, C.; Fleet, G. W. J. Tetrahedron 1999, 55, 4489-4500.

19. Myers, R. W.; Lee, Y. C. Carbohydr. Res. 1986, 154, 145163.

20. Witczak, Z. J. J. Carbohydr. Chem. 1984, 3, 359-380.

21. De las Heras, F. G.; Fernandez-Resa, P. J. Chem. Soc., Perkin Trans. 1 1982, 4, 903-907.

22. Betaneli, V. I.; Ovchinnikov, M. V.; Bachkinovskii, L. V.; Kochetkov, N. K. Carbohydr. Res. 1979, 76, 252-256.

23. Bochkov, A. F.; Kochetkov, N. K. Carbohydr. Res. 1975 39, 355-357.

24. Kochetkov, N. K. Tetrahedron 1987, 43, 2389-2436.

25. Sicherl, F.; Wittmann, V. Angew. Chem., Int. Ed. 2005, 44, 2096-2099.

26. Gruner, S. A. W.; Truffault, V.; Voll, G.; Locardi, E.; Stöckle, M.; Kessler, H. Chem. Eur. J. 2002, 8, 4365-4376.

27. Semenov, V. V.; Bogdanov, V. S.; El'yanov, B. S.; Mel'nikova, L. G.; Shevelev, S. A.; Zhulin, V. M.; Fainzil'berg, A. A. Khim. Geterotsikl. Soedin. 1982, $1118-1124$.

28. Narisada, M.; Terui, Y.; Yamakawa, M.; Watanabe, F.; Ohtani, M.; Miyazaki, H. J. Org. Chem. 1985, 50, 2794 2796.

29. Vogel, C.; Morales Torres, G.; Reinke, H.; Michalik, D.; Voss, A. Carbohydr. Res. 2007, 342, 520-528.

30. Lemieux, R. U.; Morgan, A. R. Can. J. Chem. 1965, 43, $2199-2204$.

31. Utimoto, K.; Wakabayashi, Y.; Horiie, T.; Inoue, M.; Shishiyama, Y.; Obayashi, M.; Nozaki, H. Tetrahedron 1983, 39, 967-973. 
32. Liang, C. W.; Kim, M. J.; Jeong, L. S.; Chun, M. W.. Nucleosides Nucleotides Nucleic Acids 2003, 22, 2039-2048.

33. Hirschmann, R.; Nicolaou, K. C.; Pietranico, S.; Salvino, J.; Leahy, E. M.; Sprengeler, P. A.; Furst, G.; Smith, A. B., III; Strader, C. D.; Cascieri, M. A.; Candelore, M. R.;
Donaldson, C.; Vale, W.; Maechler, L. J. Am. Chem. Soc. 1992, 114, 9217-9218.

34. Armarego, W. L. F.; Perrin, D. D. Purification of Laboratory Chemicals, 4th ed.; Pergamon Press: Oxford, 2002. 